\title{
A Three Dimensional Large Deformation Meshfree Method for Arbitrary Evolving Cracks
}

\author{
Rabczuk T. Belytschko T. \\ Department of Mechanical Engineering, Northwestern University, Evanston, IL \\ 60208-311, U.S.A
}

\begin{abstract}
A new approach for modelling discrete cracks in meshfree particle methods in three dimensions is described. The cracks can be arbitrarily oriented, but their growth is represented discretely by activation of crack surfaces at individual particles, so no representation of the crack's topology is needed. The crack is modelled by a local enrichment of the test and trial functions with a sign function (a variant of the Heaviside step function), so that the discontinuities are along the direction of the crack. The discontinuity consists of cylindrical planes centered at the particles. The method is formulated for large deformations and arbitrary nonlinear and ratedependent materials; cohesive laws govern the traction-crack opening relations. To reduce computational cost and since more accuracy around the crack tip is needed to obtain adequate results, h-adaptivity is incorporated in the method. The model is applied to several three dimensional problems, some of which are compared to experimental data.
\end{abstract}

Key words: meshfree methods, cohesive crack model, dynamic fracture

\section{INTRODUCTION}

A recent paper [36] proposed a 'cracking particle' method for modelling arbitrary crack propagation in two dimensions. The essential idea of this meshfree method is to introduce discontinuities at particles around the surface of the crack. A continuous crack is thus represented by a contiguous set of cracked particles. There is consequently no need to construct a representation of the crack surface, which leads to a method of compelling simplicity.

Email address: t-belytschko@northwestern.edu (Rabczuk T.). 
The complexity of the method is even less than that of the interelement separation models and it is significantly less complex than the extended finite element method (XFEM). In the former, the cracks can be modelled only along element interfaces in the mesh, see Xu and Needleman [48], Camacho and Ortiz [14], Ortiz and Pandolfi [34] and Zhou and Molinari [50]. Furthermore, the interelement crack method can result in an overestimate of the fracture energy when the actual crack paths are not coincident with element edges and the results of interelement crack methods depend significantly on mesh refinement, see Falk et al. [21]. This difficulty has been alleviated by adding randomness to the strength, as in Zhou and Molinari [50], though such corrections are not yet understood. In contrast to interelement methods, the proposed method can treat arbitrary crack paths, but whereas in interelement crack methods the crack path is continuous, for the proposed method the cracks are discontinuous.

In the extended finite element method, (which has been applied to static problems in two and three dimensions, [22, 30, 31] and to dynamic problems, Belytschko et al. [4]), an explicit representation of the crack surface is needed. This is provided by level sets in Gravouil et al. [22] and Belytschko et al. [4]. Although level set methods are very powerful for tracking surfaces, they introduce some complexity; for example in the treatment of crack branching, another level set needs to be introduced whenever a crack branches.

Previous meshfree methods for the modelling of cracks (Belytschko and Tabbara [11], Lu et al. [29], Belytschko and $\mathrm{Lu}$ [8], Belytschko et al. [9] and Krysl and Belytschko [25]) also used explicit representations of the crack. In the three-dimensional method in [25], surface models were constructed from 3 -node triangular facets.

The embedded discontinuity methods (Belytschko et al. [5], Armero and Garikipati [1], Samaniego et al. [42]) do not require a surface representation. However, their effectiveness in crack dynamics has still not been verified and these methods require the crack to propagate one element at a time.

In this paper, we study the applicability of the method proposed in [36] to brittle fracture in three dimensions. We use an adaptive meshfree method in a structured (Cartesian) arrangement of particles. Although this appears at first to be dissonant with the philosophy of meshfree methods, we take advantage of the versatility of meshfree methods in modelling discontinuities (i.e. cracks) and in implementing h-adaptivity; we show the latter is quite important in obtaining accurate solutions by this method.

We also introduce in this paper a method for transitioning from Lagrangian to Eulerian kernels. Belytschko et al. [6] have shown previously that Eulerian kernels tend to exhibit spurious instabilities in stretching. For a hyperelastic 
material in two-dimensional deformation with any positive principal strain, a particle method is unstable in tension; this has been verified for other materials. This behavior was first noted in one dimension by Swegle and Hicks [44]. This behavior of the Eulerian kernel leads to spurious fracture when these particle methods are applied to solids. In Rabczuk and Belytschko [36], it was shown that a Lagrangian kernel eliminates this spurious instability.

Therefore, we use a Lagrangian kernel for the initial stages of our simulations. However, for large deformations and contact-impact, Lagrangian kernels are not effective. Therefore, we have devised a method for switching to an Eulerian kernel at the nodes that are cracked.

The article is arranged as follows: The governing equations are given in section 2. Then, the method for representing cracks is reviewed in section 3. Within this section we will focus on modelling material fracture using Lagrangian kernels combined with Eulerian kernels. An adaptive scheme is proposed afterwards. The constitutive and cohesive models used are explained in section 4 as well as criteria for switching from a continuum to a discontinuum. We will address the issue of crack closure and cyclic loading in this section. In section 5 we describe the application of the method to several static and dynamic problems: a mode I crack problem with analytic solution, a penny-shaped crack problem, a notched concrete beam under four-point-bending, a notched concrete beam under four-point-bending with non-planar crack growth, failure of a reinforced concrete beam, 3D crack branching problem, impact problems and concrete slabs under explosive loading. In the latter two examples penetration as well as perforation occurs. The results are compared to experimental data or other results from the literature.

\section{GOVERNING EQUATIONS}

We consider a body $\Omega$ in $\Re^{3}$ with boundary $\Gamma$; their images in the initial state are the open set $\Omega_{0}$ and the boundary $\Gamma_{0}$, respectively. $\Gamma_{0}$ is the boundary with $\Gamma_{0}=\Gamma_{0}^{c} \cup \Gamma_{0}^{t} \cup \Gamma_{0}^{u}$ and $\Gamma_{0}^{c} \cap \Gamma_{0}^{t} \cap \Gamma_{0}^{u}=\varnothing$ where $\Gamma_{0}^{c}$ is the crack surface, $\Gamma_{0}^{t}$ the prescribed traction surface and $\Gamma_{0}^{u}$ the prescribed displacement surface. The initial state will also serve as the reference state. The strong form of the linear momentum equation in a total Lagrangian description is:

$$
\nabla_{0} \cdot \mathbf{P}+\varrho_{0} \mathbf{b}=\varrho_{0} \ddot{\mathbf{u}} \text { in } \Omega_{0} \backslash \Gamma_{0}^{c}
$$

and the boundary conditions are

$$
\mathbf{n}_{0} \cdot \mathbf{P}=\overline{\mathbf{t}}_{0} \text { on } \Gamma_{0}^{t}
$$




$$
\begin{array}{r}
\mathbf{u}=\overline{\mathbf{u}} \text { on } \Gamma_{0}^{u} \\
\mathbf{n}_{0} \cdot \mathbf{P}^{-}=\mathbf{n}_{0} \cdot \mathbf{P}^{+}=\mathbf{t}_{c 0} \text { on } \Gamma_{0}^{c}
\end{array}
$$

where $\mathbf{P}$ is the nominal stress (note that $\mathbf{P}$ is the transpose of the first PiolaKirchhoff tensor), $\nabla_{0}$ is the divergence in the material coordinate system, $\varrho_{0}$ is the initial density, $\mathbf{b}$ are the body forces, $\mathbf{u}$ and $\ddot{\mathbf{u}}$ are the displacements and accelerations (a superimposed dot denotes a material time derivative or a time derivative depending on the context), respectively; $\mathbf{n}_{0}$ is the normal to the boundary in the initial configuration, $\overline{\mathbf{u}}$ and $\overline{\mathbf{t}}$ denote the prescribed displacements and tractions, respectively and $\mathbf{t}_{c 0}$ are the cohesive forces across the crack.

We will also use the strong form of the linear momentum equation in the updated Lagrangian description

$$
\nabla \cdot \boldsymbol{\sigma}+\varrho \mathbf{b}=\varrho \ddot{\mathbf{u}} \text { in } \Omega \backslash \Gamma^{c}
$$

where the boundary conditions are

$$
\begin{array}{r}
\mathbf{n} \cdot \boldsymbol{\sigma}=\overline{\mathbf{t}} \text { on } \Gamma^{t} \\
\mathbf{u}=\overline{\mathbf{u}} \text { on } \Gamma^{u} \\
\mathbf{n} \cdot \boldsymbol{\sigma}^{-}=\mathbf{n} \cdot \boldsymbol{\sigma}^{+}=\mathbf{t}_{c} \text { on } \Gamma^{c}
\end{array}
$$

where $\boldsymbol{\sigma}$ is the Cauchy stress, $\varrho$ is the current density and $\mathbf{n}$ is the normal to the relevant surface in the current configuration. In the following sections we will give the discrete equations in the total Lagrangian description, but some terms are evaluated in updated Lagrangian format when convenient.

\section{THE EFG-P METHOD}

\subsection{Displacement field}

Consider a displacement field which is continuous in the entire domain except at the cracks where a discontinuity occurs in the displacements. To describe this discontinuity, the displacement is decomposed into continuous and discontinuous parts as in $[3,19,30]$ :

$$
\mathbf{u}(\mathbf{X}, t)=\mathbf{u}^{\text {cont }}(\mathbf{X}, t)+\mathbf{u}^{e n r}(\mathbf{X}, t)
$$

where $\mathbf{X}$ are the material coordinates, $t$ is the time, $\mathbf{u}^{\text {cont }}$ denotes the continuous displacement and $\mathbf{u}^{e n r}$ the discontinuous part, which is also called the 
enrichment.

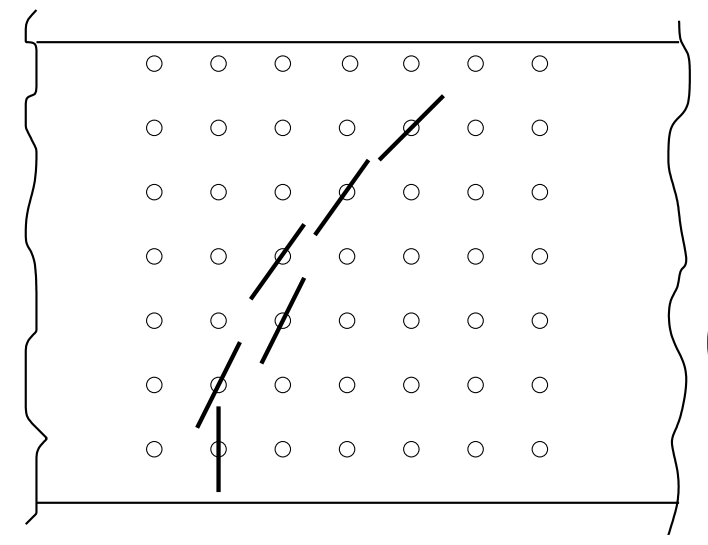

a)

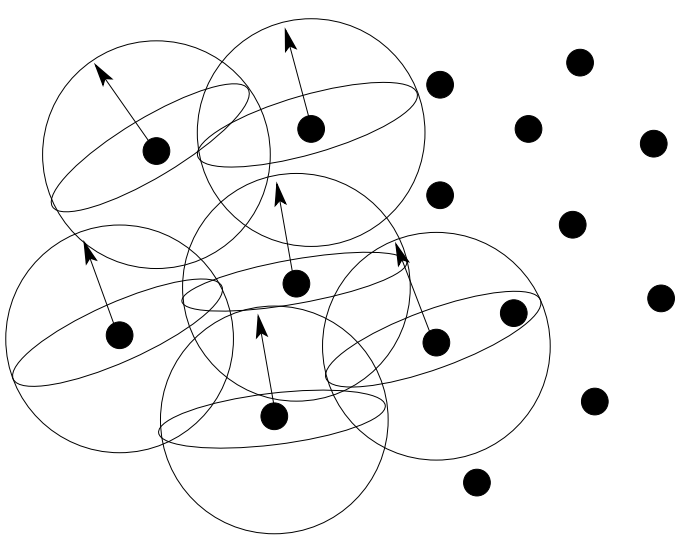

(b)

Fig. 1. Schematic of the crack model for two and three dimensions

The crack is modelled by a set of discrete cracks as shown in figure 1 . These discrete cracks are restricted to be centered on the particles, i.e. each crack plane always passes through a particle. Since the crack geometry is described by the set of cracked particles, we do not have to provide a representation for the geometry of the crack.

Let $\mathcal{N}$ be the total set of nodes in the model and $\mathcal{N}_{c}$ the set of cracked nodes. To model the discontinuous part of the displacement, the test and trial functions are enriched with sign functions which are parametrized by $\delta \mathbf{q}_{I}$ and $\mathbf{q}_{I}$, respectively. Only cracked nodes are enriched. We will describe the criteria for "cracking" the particles subsequently.

The test and trial functions are

$$
\begin{aligned}
& \mathbf{u}^{h}(\mathbf{X}, t)=\sum_{I \in \mathcal{N}} \hat{\Phi}_{I}(\mathbf{X}) \mathbf{u}_{I}(t)+\sum_{I \in \mathcal{N}_{c}} \Psi_{I}(\mathbf{X}) S\left(f_{I}(\mathbf{X})\right) \mathbf{q}_{I}(t) \\
& \delta \mathbf{u}^{h}(\mathbf{X})=\sum_{I \in \mathcal{N}} \Phi_{I}(\mathbf{X}) \delta \mathbf{u}_{I}+\sum_{I \in \mathcal{N}_{c}} \Psi_{I}(\mathbf{X}) S\left(f_{I}(\mathbf{X})\right) \delta \mathbf{q}_{I}
\end{aligned}
$$

where $f_{I}(\mathbf{X})$ is given by

$$
f_{I}(\mathbf{X})=\mathbf{n}_{0} \cdot\left(\mathbf{X}-\mathbf{X}_{I}\right)
$$

where $\mathbf{n}_{0}$ is the normal to the crack in the reference configuration and $\mathbf{u}_{I}$ are the particle displacements. The normal in the initial configuration $\mathbf{n}_{0}$ is found by $\mathbf{n}$ from Nanson's law

$$
\mathbf{n}_{0}=J^{-1} \mathbf{n}\left(t_{c r}\right) \cdot \mathbf{F}\left(\mathbf{X}_{I}, t_{c r}\right)
$$


where $t_{c r}$ is the time at which the particle cracks. The current normal can then be found from $\mathbf{n}_{0}$ by a rearrangement of the above equation.

The sign function $S(\xi)$ is defined as:

$$
S(\xi)=\left\{\begin{array}{c}
1 \quad \forall \xi>0 \\
-1 \quad \forall \xi<0
\end{array}\right.
$$

In general, different shape functions can be used for the continuous part, $\Phi_{I}(\mathbf{X})$, and discontinuous part, $\Psi_{I}(\mathbf{X})$, see eq. (11) and (10). Here, we will employ the same shape functions. Note that we will use a Petrov-Galerkin method, so the test and trial functions differ. The support of the cracked particle is equal to the support size of the other shape functions. If the discretization is refined, they are both decreased in size so that the number of initial neighbors are the same for all discretizations.

The gradient of the test functions with respect to the material coordinates is given by

$$
\begin{aligned}
\nabla_{0} \delta \mathbf{u}(\mathbf{X}) & =\sum_{I \in \mathcal{N}} \nabla_{0} \Phi_{I}(\mathbf{X}) \otimes \delta \mathbf{u}_{I}+\sum_{I \in \mathcal{N}_{c}} \nabla_{0} \Psi_{I}(\mathbf{X}) S\left(f_{I}(\mathbf{X})\right) \otimes \delta \mathbf{q}_{I} \\
& +\sum_{I \in \mathcal{N}_{c}} \Psi_{I}(\mathbf{X}) \nabla_{0} S\left(f_{I}(\mathbf{X})\right) \otimes \delta \mathbf{q}_{I}
\end{aligned}
$$

The last term on the RHS of eq. (15) is dropped since $\Omega_{0}$ is treated as an open set with the crack as part of the boundary and $\nabla_{0} S\left(f_{I}(\mathbf{X})\right)$ is nonzero only on $\Gamma_{c}^{0}$. The trial functions have an identical structure.

\subsection{Weak form and discretization}

We make the approximation that the crack surface can be represented by a set of disconnected surfaces $\Gamma_{0 I}^{c}$ so that

$$
\Gamma_{0}^{c}=\sum_{I \in \mathcal{N}_{c}} \Gamma_{0 I}^{c}
$$

where $\Gamma_{0 I}^{c}$ is the crack surface for cracked particle $I$; it denotes a single surface in the reference configuration but in the current configuration $\Gamma_{0 I}^{c}$ become two surfaces. While $\Gamma_{0}^{u}$ and $\Gamma_{0}^{t}$ are time invariant, $\Gamma_{0}^{c}$ changes with time as the cracks grow. 


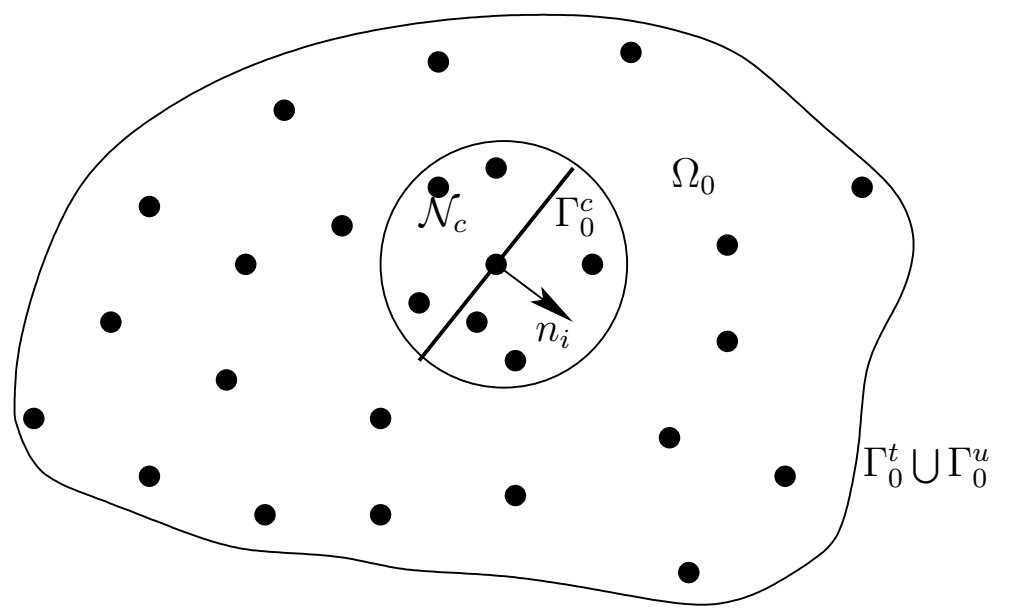

Fig. 2. Domain with a single cracked particle

The weak form of the momentum equation is the standard principle of virtual work (see e.g. Belytschko et al. [7]): find $\mathbf{u} \in \mathcal{V}$ such that

$$
\delta W=\delta W_{\text {int }}-\delta W_{\text {ext }}+\delta W_{\text {kin }}=0 \quad \forall \delta \mathbf{u} \in \mathcal{V}_{0}
$$

where

$$
\begin{aligned}
& \mathcal{V}=\left\{\mathbf{u}(\cdot, t) \mid \mathbf{u}(\cdot, t) \in H^{1}, \mathbf{u}(\cdot, t) \text { discontinuous on } \Gamma_{0}^{c} \mathbf{u}(\cdot, t)=\overline{\mathbf{u}}(t) \text { on } \Gamma_{0}^{u}\right\} \\
& \mathcal{V}_{0}=\left\{\delta \mathbf{u} \mid \delta \mathbf{u} \in \mathcal{V}, \delta \mathbf{u}=0 \quad \text { on } \Gamma_{u}\right\} \\
& \delta W_{\text {int }}=\int_{\Omega_{0} \backslash \Gamma_{0}^{c}}\left(\nabla_{0} \otimes \delta \mathbf{u}\right)^{T}: \mathbf{P} d \Omega_{0} \\
& \delta W_{\text {ext }}=\int_{\Omega_{0} \backslash \Gamma_{0}^{c}} \varrho_{0} \mathbf{b} \cdot \delta \mathbf{u} d \Omega_{0}+\int_{\Gamma_{0}^{t}} \overline{\mathbf{t}}_{0} \cdot \delta \mathbf{u} d \Gamma_{0}+\int_{\Gamma^{c}} \mathbf{t}_{c} \cdot \llbracket \delta \mathbf{u} \rrbracket d \Gamma \\
& \delta W_{k i n}=\int_{\Omega_{0} \backslash \Gamma_{0}^{c}} \varrho_{0} \delta \mathbf{u} \cdot \ddot{\mathbf{u}} d \Omega_{0}
\end{aligned}
$$

where the prefix $\delta$ denotes the test function and $W_{\text {ext }}$ is the external energy, $W_{\text {int }}$ the internal energy and $W_{k i n}$ the kinetic energy. The current normal for each crack segment is obtained by Nanson's law.

A Petrov-Galerkin method (see also Rabczuk et al. [38]) with linear complete MLS shape functions as test functions is used; the trial functions are Shepard functions but their gradients are obtained by the Krongauz and Belytschko [24] correction since they do not satisfy the reproducing conditions for linear 
polynomials, which is essential for convergence (the Randles-Libersky correction [40] is similar, see also Bonet and Kulasegaram [12], Bonet and Lok [13] and Vila [46]).

The trial functions are constructed as usual for moving least square approximations by minimizing:

$$
e(\mathbf{a})=\sum_{J \in \mathcal{S}} w\left(\mathbf{X}-\mathbf{X}_{J}\right)\left(\mathbf{p}^{T}\left(\mathbf{X}_{I}\right) \mathbf{a}(\mathbf{X}, t)-\mathbf{u}_{I}(t)\right)
$$

where $w$ is a weight function of compact support, $\mathbf{p}(\mathbf{X})$ is a polynomial basis (we used a linear basis, so $\mathbf{p}^{T}=\left[\begin{array}{llll}1 & X & Y & Z\end{array}\right]$ ), a are unknown parameters and $\mathbf{u}_{I}$ are the nodal values of the function to be approximated. We used the cubic spline as weight function.

Finally, the discrete equations are

$$
\mathbf{M}_{I J} \cdot \ddot{\mathbf{u}}_{J}=\mathbf{f}_{I}^{e x t}-\mathbf{f}_{I}^{\text {int }}
$$

(see e.g. Belytschko et al. [7]). In the above, $\mathbf{M}_{I J}$ is the mass matrix given in Rabczuk and Belytschko [36]. The mass matrix $\mathbf{M}_{I J}$ was diagonalized by a row-sum technique on the uncracked particles, but the full consistent mass was used on the cracked particles.

The vector $\mathbf{f}_{I}^{e x t}$ is the external nodal force vector and given by

$$
\begin{aligned}
\mathbf{f}_{I}^{e x t} & =\left\{\begin{array}{c}
\mathbf{f}_{I}^{u, e x t} \\
\mathbf{f}_{I}^{q, e x t}
\end{array}\right\} \\
\mathbf{f}_{I}^{u, e x t} & =\int_{\Omega_{0} \backslash \Gamma_{0}^{c}} \varrho_{0} \mathbf{b} \Phi_{I}(\mathbf{X}) d \Omega_{0}+\int_{\Gamma_{0}^{t}} \overline{\mathbf{t}}_{0} \Phi_{I}(\mathbf{X}) d \Gamma_{0} \\
\mathbf{f}_{I}^{q, e x t} & =\int_{\Gamma^{c}}\left(\mathbf{t}_{c} \Psi_{I}(\mathbf{X}) \llbracket S\left(f_{I}(\mathbf{X})\right) \rrbracket+\alpha \Psi_{I}(\mathbf{X}) \llbracket S\left(f_{I}(\mathbf{X})\right) \rrbracket \dot{\mathbf{u}}\right) d \Gamma \\
& +\int_{\Omega_{0} \backslash \Gamma_{0}^{c}} \varrho_{0} \mathbf{b} \Psi_{I}(\mathbf{X}) S\left(f_{I}(\mathbf{X})\right) d \Omega_{0}+\int_{\Gamma_{0}^{t}} \overline{\mathbf{t}}_{0} \Psi_{I}(\mathbf{X}) S\left(f_{I}(\mathbf{X})\right) d \Gamma_{0}
\end{aligned}
$$

The vector $\mathbf{f}_{I}^{\text {int }}$ is the internal nodal force vector and given by 


$$
\begin{aligned}
& \mathbf{f}_{I}^{i n t}=\left\{\begin{array}{c}
\mathbf{f}_{I}^{u, i n t} \\
\mathbf{f}_{I}^{q, i n t}
\end{array}\right\} \\
& \mathbf{f}_{I}^{u, i n t}=\int_{\Omega_{0} \backslash \Gamma_{0}^{c}} \nabla_{0} \Phi_{I}(\mathbf{X}) \cdot \mathbf{P}(\mathbf{X}) d \Omega_{0} \\
& \mathbf{f}_{I}^{q, i n t}=\int_{\Omega_{0} \backslash \Gamma_{0}^{c}} \nabla_{0} \Psi_{I}(\mathbf{X}) \cdot \mathbf{P}(\mathbf{X}) S\left(f_{I}(\mathbf{X})\right) d \Omega_{0}
\end{aligned}
$$

\subsection{Eulerian and Lagrangian kernel for modelling material fracture}

In most SPH procedures (see eg. Randles and Libersky [40, 41], Dilts [17, 18]), an Eulerian kernel is used:

$$
w_{J}(\mathbf{x})=w\left(\mathbf{x}-\mathbf{x}_{J}(t), h(\mathbf{x}, t)\right)
$$

The Eulerian kernel is expressed in terms of spatial coordinates. The radius $h$ of the support depends on the spatial coordinates and can vary in time.

Belytschko et al. [6] have shown that particle discretizations of solids with an Eulerian kernel lead to a distortion of the stable domain of the material in stress space; the tensile instability first identified in Swegle and Hicks [44] is one manifestion of this distortion.

The Lagrangian kernel is expressed in terms of material coordinates, so:

$$
w_{J}(\mathbf{X})=w\left(\mathbf{X}-\mathbf{X}_{J}, h_{0}\right)
$$

For Lagrangian kernels, the neighbors do not change during the course of the simulation, but the domain of influence in the current configuration becomes distorted with time. A Lagrangian kernel provides a more consistent procedure when simulating material fracture because instabilities will not occur due to numerical artifacts. Hence, in [36], we used a Lagrangian kernel to simulate material fracture. However, this limits the magnitude of the distortions that can be treated since in the current configuration the domain of influence of a Lagrangian kernel can become extremely distorted after a body cracks. Therefore, we here use a method that combines Lagrangian and Eulerian kernels. This is essential when modelling fracture and fragmentation as in some of the examples reported here, see e.g. section 5.8. 
In calculations with large deformations, we start with a Lagrangian kernel. After introducing a discontinuity, we switch to an Eulerian kernel when the particles have separated or if the cohesive tractions have decayed to zero. At this point, the enrichment is removed and the particle is split into two particles. Kinematic values such as velocity are assigned to the corresponding particle according to the jump in the velocity fileld. By using initially a Lagrangian kernel, we ensure that material fracture occurs physically and not due to numerical artifacts. For very large deformations, the Eulerian kernel ensures that the solution is stable and allows large separations.

In the blending domain between Lagrangian and Eulerian kernels, we proceed as follows: The kernels that are not cracked are kept Lagrangian but exclude particles on the opposite side of the crack from the domain of influence. In other words, we check if the opposite cracked particles are not in the domain of influence in the current configuration of the blended particles. If they do, they are removed from the domain of influence of the blended particles. This is a straightforward check since all neighbors and connectivities are known. This way we ensure stable results in the blending region. We will show that this approach gives stable results while results obtained by computations with pure Lagrangian or Eulerian kernels are unstable.

\subsection{Integration and particle masses and volumes}

The integrals in (25) and (27) are evaluated by stress point integration similar to Rabczuk and Belytschko [36]. The particle masses and volumes are obtained via a Voronoi tesselation.

In the 3D procedure, we use a structured particle arrangement: 8 particles are arranged so they form a cuboid. A stress point is placed in the middle of the cuboid as shown in figure $3 \mathrm{a}$. The cuboid is then subdivided into 12 tetrahedra for computation of the volumes.

Once the coordinates of the particles and stress points are known, the tetrahedral volumes can easily be computed. The volumes are distributed equally between the particles and stress points. An excellent overview on triangulation and Voronoi procedures with algorithmic details can be found in Loehner [27] and Carey [15]. Note, that the particle masses and volumes have to be calculated only once at the beginning of the computation for particles that do not crack; i.e. as long as the kernels are Lagrangian. Any integral, such as (25) or (27) is then calculated by

$$
\int_{\Omega_{0}} \Phi_{I}(\mathbf{X}) g(\mathbf{X}) d \Omega_{0}=\sum_{J \in \mathcal{N}_{I}} \Phi_{I}\left(\mathbf{X}_{J}\right) g\left(\mathbf{X}_{J}\right) V_{J}^{0}
$$


where $V_{J}^{0}$ is the Voronoi volume of node $J$ and $\mathcal{N}_{I}$ is the set of nodes in the support of $\Phi_{I}(\mathbf{X})$.

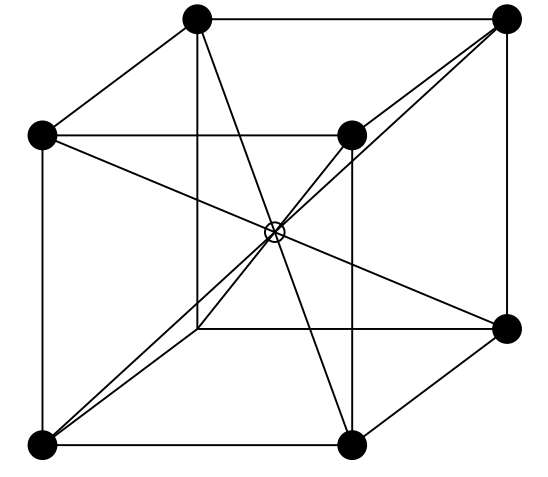

A
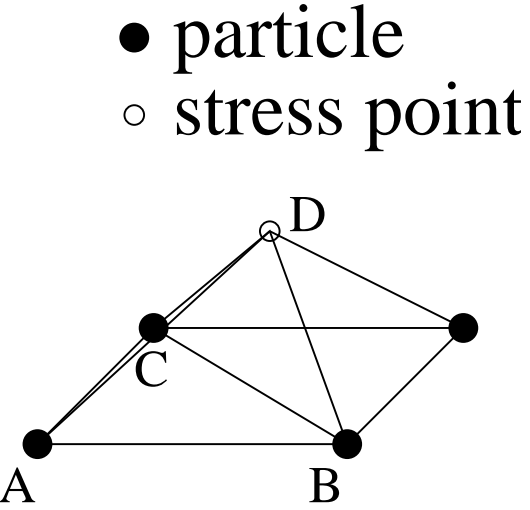

Fig. 3. Stress point arrangements in 3D using a structured particle arrangement

\subsection{Adaptivity}

In some 3D applications, the EFG-P method needs many particles to obtain acceptable results. Therefore, we have introduced an adaptive scheme that allows us to start with a coarse discretization and only those parts of the structure where cracking occurs are refined. 2D adaptive schemes for particle methods with stress points or nodal integration have been presented by You et al. [49], Lu and Chen [28].

We based our adaptive scheme on an adaptive scheme for structured particle arrangements, see Rabczuk and Belytschko [37]. The criterion for adding particles adaptively is based on an estimate of the approximation error. Particles are added where large strain gradients occur, which generally indicates where the crack will be. For further details on the error estimation procedure, see [37].

One difficulty of adaptive schemes in particle methods with stress points is the reconstruction of the Voronoi cells and the recomputation of the particle and stress point masses. Since construction of the Voronoi cells is computationally expensive, reconstruction of the Voronoi cells in the entire domain should be avoided. Reconstruction of the Voronoi cells is only needed in the neighborhood of refinement.

Similar to finite elements, connectivity arrays exist between the cuboids (and their sub-geometries) and the particles. Therefore, efficient data structures (such as N-trees instead of linked lists) are preferable. Details about such data structures and effective programming can be found in Loehner [27] and Carey [15]. 
We applied the Devloo and Oden rule [16] to assure a smooth transition between the coarse and fine discretizations. A new support size $h$ has to be assigned for particles in the refined and transition region. A smooth transition is created by averaging the support size for particles in the transition surface by $h_{K}=0.5\left(h_{I}+h_{J}\right)$, where the subscript $K$ denote transition particles, $I$ denote particles in the refined domain and $J$ are the particles in their neighbor region. The new data has to be mapped on the adaptively added particles and stress points. This is done by an MLS interpolation. Further details are given in Rabczuk and Belytschko [37].

\subsection{Contact}

In some of the examples contact occurs. In particle methods based on Eulerian kernels, contact of two bodies can be handled quite easily; contact conditions are enforced naturally if particles of two different bodies fall in their mutual domain of influence. Care has to be taken that only compressive forces and no tensile forces are transmitted. In special cases (e.g. in contact detonations), contact can be modelled via the kernel function even with Lagrangian kernels. However, in general, contact detection algorithms are necessary if Lagrangian kernels are used. A simple and robust contact algorithm is the pinball algorithm, see Belytschko and Neal [10].

\section{CONSTITUTIVE AND COHESIVE MODELS}

The constitutive/cohesive model consists of three ingredients. The starting point of the constitutive model is a continuum constitutive equation. We used a model described in Rabczuk and Eibl [39] which is suitable for concrete. We also used the Lemaitre model [26] and Rankine models. The models will be briefly reviewed in the following section.

The second ingredient is a criterion for when to introduce the discontinuity. A discrete crack is introduced whenever a criterion for loss of continuity is met at a particle; the traction across the crack is then governed by the third ingredient, a traction-crack opening law, often called a cohesive law. Several criteria for loss of continuity were studied which will be described subsequently. The cohesive models will be described in section 4.3. 

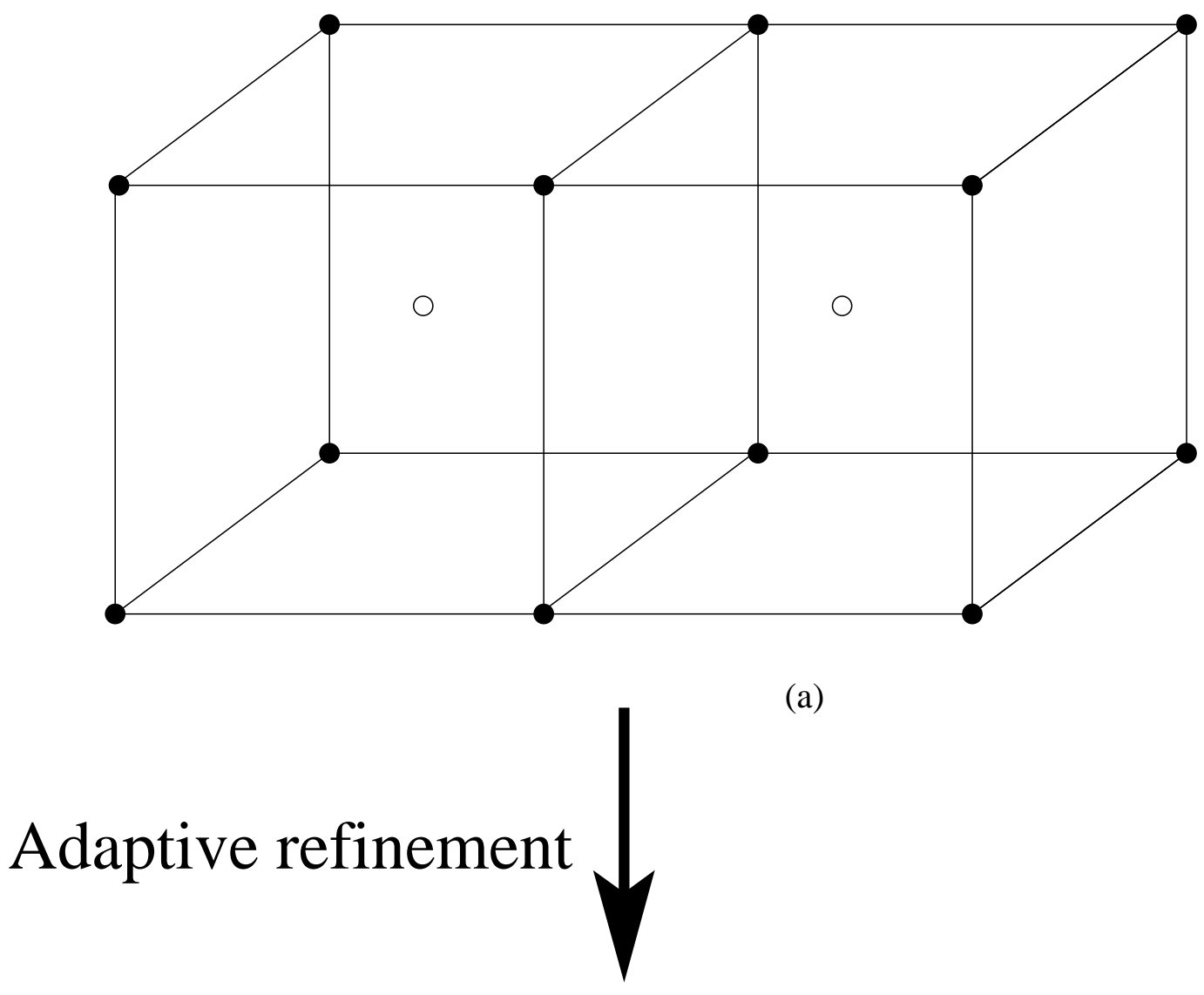

(a)

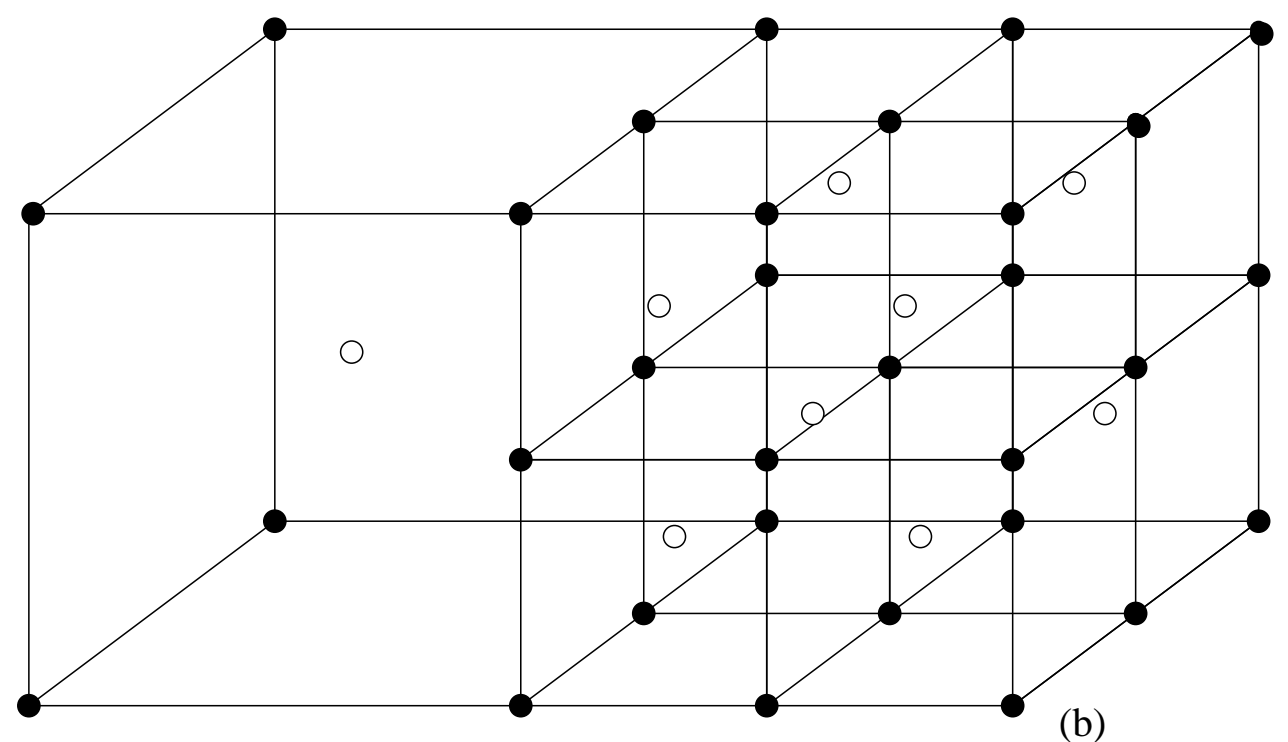

Fig. 4. Adaptivity in 3D showing initial mesh and partially refined mesh 


\subsection{Continuum models}

\subsubsection{The Lemaitre model}

The Lemaitre model [26] was developed for brittle materials such as concrete and is an elastic model with isotropic damage. The stress-strain relation is given by

$$
\boldsymbol{\sigma}=(1-D) \mathbf{C} \boldsymbol{\epsilon}
$$

where $D$ is a damage variable which increases from 0 to a maximum of 1 . The damage evolution depends on the effective strain $\bar{\epsilon}$ :

$$
D(\bar{\epsilon})=1-(1-A) \epsilon_{D_{0}} \bar{\epsilon}^{-1}-A e^{-B\left(\bar{\epsilon}-\epsilon_{D_{0}}\right)}
$$

with

$$
\bar{\epsilon}=\sqrt{\sum_{i=1}^{3} \epsilon_{i}^{2} H\left(\epsilon_{i}\right)}
$$

where $\epsilon_{i}$ are the principal strains and with

$$
\begin{aligned}
& H(x)=1 \text { if } x>0 \\
& H(x)=0 \text { if } x<0
\end{aligned}
$$

$A, B$ and $\epsilon_{D_{0}}$ are material parameters. We mainly used the loss of hyperbolicity criterion for introducing a discrete crack when applying the Lemaitre model.

\subsubsection{Viscous damage model}

A strain rate dependent damage-plasticity model for concrete proposed by Rabczuk and Eibl [39] is used for concrete. For small elastic strains, the stressstrain relation is given by

$$
\boldsymbol{\sigma}=\left(1-\left(D_{s t}-D_{d y n}\right)\right) \gamma \mathbf{C}_{s} \boldsymbol{\epsilon}_{e}
$$

where $D_{s t}$ is the static damage which is decayed by a dynamic damage $D_{d y n}$, $\gamma$ is a function which takes high hydrostatic stress states into account and $\boldsymbol{\epsilon}_{e}$ is the elastic strain tensor. The damage surface is given in the strain space by

$$
F_{d}=c_{1} J_{2}^{e}+\kappa_{d}\left(c_{2} \sqrt{J_{2}^{e}}+c_{3} \epsilon_{e, \max }^{(\alpha)}+c_{4} I_{1}^{e}\right)-\kappa_{d}^{2}=0
$$


where $c_{1}$ to $c_{4}$ are parameters of the damage surface, $I_{1}^{e}$ is the first invariant of the elastic strain tensor, $J_{2}^{e}$ is the second invariant of $\operatorname{dev} \boldsymbol{\epsilon}_{e}$ and $\epsilon_{e, \max }^{(\alpha)}$ the maximum $\alpha-t h$ characteristic value of $\epsilon_{e}$. We used the same damage surface in tension and compression.

The damage evolution is given by

$$
\hat{D}\left(\kappa_{d}\right)=1-e^{-\left(\frac{\kappa_{d}-e_{0}}{e_{d}}\right)^{g_{d}}} \quad \kappa_{d} \geq e_{0} \quad \text { and } \quad \hat{D}\left(\kappa_{d}\right)=0 \quad \kappa_{d}<e_{0}
$$

where $e_{0}, g_{d}$ and $e_{d}$ are material parameters.

The dynamic damage evolution is introduced to take strain rate effects into account and is given by

$$
\hat{D}_{d y n}=\int_{\tau=0}^{t} \frac{\partial \hat{D}}{\partial \tau} \mathcal{H}(t-\tau) d \tau
$$

where the history function $\mathcal{H}(t-\tau)$ is given by

$$
\mathcal{H}(t-\tau)=e^{-\left(\frac{t-\tau}{\vartheta\left(\kappa_{d}^{*}\right)}\right)^{g_{h}}}
$$

with $g_{h}$ as material parameter. $\mathcal{H}$ decays monotonously from 1 to 0 . The relaxation time is defined by an empirical function of the equivalent elastic strain rate $\dot{\kappa}_{d}^{*}$ related to a reference value $\dot{\kappa}_{0}$

$$
\vartheta\left(\dot{\kappa}_{d}^{*}\right)=\vartheta_{0} \cdot \dot{\kappa}_{d}^{* c_{t 1} \ln \dot{\kappa}_{d}^{*}-c_{t 2}} \wedge \quad \dot{\kappa}_{d}^{*}=\frac{\dot{\kappa}_{d}(\tau)}{\dot{\kappa}_{0}} \quad \dot{\kappa}_{0}=1 s^{-1}
$$

with $c_{t 1}$ and $c_{t 2}$ as material parameters. With this Maxwell type relaxation the dynamic strength increase can be approximated in a wide range of strain rates. Note, that the model is implemented in rate form so that it is applicable to large deformations. More details can be found in [39].

\subsection{Cracking criteria}

\subsubsection{Loss of material stability criterion}

In many of the computations, we use as a criterion for cracking the onset of a material instability. Material stability is checked by conditions on the acoustic tensor $\mathbf{Q}=\mathbf{n} \cdot \mathbf{A} \cdot \mathbf{n}$ where $\mathbf{A}=\mathbf{C}^{t}+\boldsymbol{\sigma} \otimes \boldsymbol{\delta}$ where $\mathbf{C}^{t}$ is the tangent stiffness and $\mathbf{n}$ 
is the direction of polarization, see Belytschko et al. [7]. For a rate independent material, the momentum equation stays hyperbolic as long as the minimum eigenvalue of $\mathbf{Q}$ is nonnegative, i.e. $\min \operatorname{eig}(\mathbf{Q})>0$. In a rate-dependent material, the PDE usually does not change type and only undergoes a material instability. Since the normal $\mathbf{n}=(\cos \alpha \cos \varphi, \cos \alpha \sin \varphi, \sin \alpha)$ depends on two angles, the procedure of finding the minimum eigenvalue of $\mathbf{Q}$ can become computationally expensive. A way to compute the eigenvalues of the acoustic tensor is given by Ortiz et al. [33].

One difficulty is that four angles (only two of them are relevant, since the other two are in the same direction but of opposite sense) are obtained from this localization analysis. We choose the direction of the maximum displacement gradient in the localization direction by maximizing

$$
g=\underbrace{\max }_{l}\left(\mathbf{n}_{l}^{T} \cdot\left(\nabla \mathbf{u} \cdot \mathbf{h}_{l}\right)\right)
$$

where the normals $\mathbf{n}_{l}$ correspond to the minimum eigenvalues of $\mathbf{Q}$.

\subsubsection{Rankine criterion}

For a Rankine material, a crack is introduced when the principal tensile stress reaches the uniaxial tensile strength at a particle. The crack is initiated perpendicular to the direction of the principal tensile stress.

\subsubsection{Other failure criteria}

As mentioned in section 4.2.1, the PDE does not lose hyperbolicity if viscous constitutive models are employed. One can question why to switch to a discontinuum if the PDEs are regularized. One advantage of a discontinuous model is that the anisotropy of the material after cracking is modelled quite easily because of the introduction of the jump in the displacement field. Also the resolution of the crack is modelled in a more accurate manner. Viscous damage laws often produce a very smeared, rather wide domains of damage. By switching to a cohesive model, more realistic crack patterns can be resolved as will be shown for several examples in section 5. We note that Etse and Willam [20] have shown that after discretization, even with a viscoplastic constitutive model, hyperbolicity can be lost if a critical time step is exceeded.

Other criteria that can be used to initiate a crack can be based on state variables, e.g. damage, effective plastic strain. The difficulty is then to give the orientation of the crack. For a mode $I$ crack, the direction chosen is parallel to the principal tensile stress. 


\subsection{The cohesive model}

When the cracking criterion is met at a particle, a discrete crack is introduced by adding the enrichment (9) as illustrated in figure 1 and eq. (10). Once a particle is cracked, the tractions across the discontinuity depend on the relative displacements of the adjacent crack surfaces, i.e. the jump or magnitude of the discontinuity in the displacement across the crack surface $\Gamma_{0}^{c}$. We consider the cohesive traction to be a function of the jumps in the normal displacements and tangential displacements.

\subsubsection{Computation of the jump in the displacement}

The jump in the displacement across the crack depends only on the discontinuous part of the displacement field $\mathbf{u}^{\text {enr }}(\mathbf{X})$ and hence the enrichment parameters $\mathbf{q}_{I}$. It can be shown from (10) that

$$
\llbracket \mathbf{u}(\mathbf{X}) \rrbracket=2 \sum_{I \in \mathcal{N}_{c}} \Psi_{I}(\mathbf{X}) \mathbf{q}_{I}
$$

Let $\delta_{n}$ be the normal component of the displacement jump across the crack and $\delta_{\tau}$ be the tangential displacement jump. Then, the relative displacements can be computed as

$$
\begin{aligned}
& \delta_{n}=\llbracket \mathbf{u}(\mathbf{X}) \rrbracket_{n}=\mathbf{n} \cdot \llbracket \mathbf{u}(\mathbf{X}) \rrbracket \\
& \delta_{\tau}=\llbracket \mathbf{u}(\mathbf{X}) \rrbracket_{\tau}=\|\llbracket \mathbf{u}(\mathbf{X}) \rrbracket-(\llbracket \mathbf{u}(\mathbf{X}) \rrbracket \cdot \mathbf{n}) \mathbf{n}\|
\end{aligned}
$$

where $\llbracket \mathbf{u}(\mathbf{X}) \rrbracket_{n}$ is the projection of $\llbracket \mathbf{u}(\mathbf{X}) \rrbracket$ on the normal $\mathbf{n}$ and $\llbracket \mathbf{u}(\mathbf{X}) \rrbracket_{\tau}$ is the projection of $\llbracket \mathbf{u}(\mathbf{X}) \rrbracket$ on the plane which is perpendicular to $\mathbf{n}$.

\subsubsection{The cohesive law}

We employ linear, bilinear and exponential relationships between the traction and crack opening as shown in figure 5, similar to Ortiz and Pandolfi [34]. Unloading is linear elastic; at the transition to compression, we switch back to the continuum model, meaning we remove the additional degrees of freedom and apply the continuum constitutive model. Note that the maximum cohesive traction has to be stored to guarantee the correct fracture energy at crack reopening. Another possibility to avoid interpenetration would be the penalty method. 


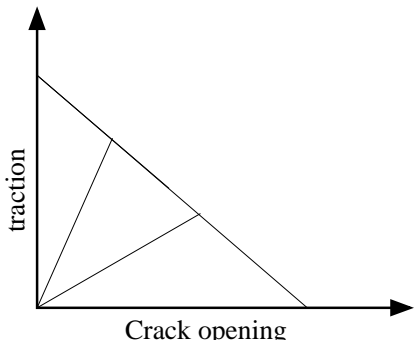

a)

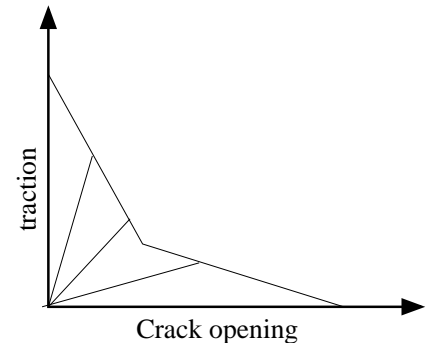

b)

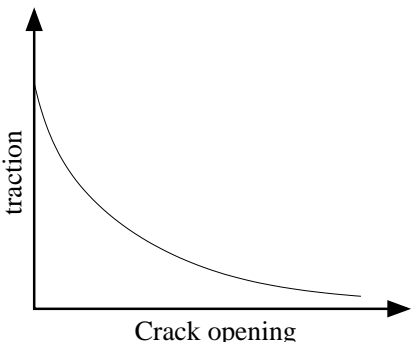

c)

Fig. 5. Cohesive models: a) Linear models, b) Bilinear models, c) Exponential models

The area under the cohesive curve, figure 5, corresponds to the fracture energy $G_{f}$. For quasi static loading conditions, the fracture energy is well documented for many materials. Experiments by e.g. Schuler [43] have shown that the fracture energy increases under dynamic loading conditions. In our dynamic computations, we take this increased fracture energy into account by increasing the dynamic fracture energy proportionally to the dynamic failure strength. Accordingly, the area under the cohesive curve is increased. A general expression of the fracture energy with respect to the strain rate is desirable but due to lack of experimental data difficult to derive.

\section{RESULTS}

\subsection{Mode I crack}

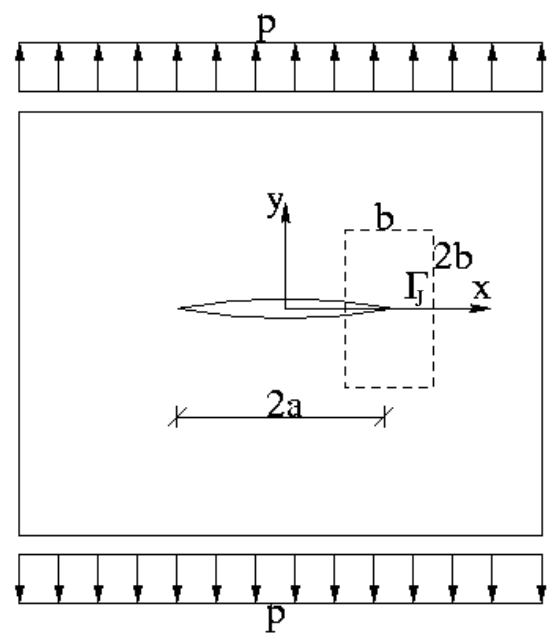

Fig. 6. The mode I crack problem

Consider the mode I crack problem illustrated in figure 6. Plane strain conditions and linear elastic material behavior are assumed. Convergence is studied 
in terms of the relative error in the energy:

$$
\| \text { err } \|_{\text {energy }}=\frac{\left\|\mathbf{u}_{h}-\mathbf{u}_{\text {analytic }}\right\|_{\text {energy }}}{\left\|\mathbf{u}_{\text {analytic }}\right\|_{\text {energy }}}
$$

with

$$
\|\mathbf{u}\|_{\text {energy }}=\left(\int_{\Omega_{0}} \mathbf{E}^{T}(\mathbf{u}): \mathbf{C}: \mathbf{E}(\mathbf{u}) d \Omega_{0}\right)^{1 / 2}
$$

where $\mathbf{E}$ is the Green strain.

The stress intensity factor $K_{I}$ is computed using a domain around the crack tip. The cohesive tractions are set to zero and we proceed as explained in section 3.3. We tested this approach for linear basis polynomials. Figure 7a shows the error in the energy norm. Figure $7 \mathrm{~b}$ shows the computed stress intensity factor compared to the analytical solution. Considering the simplicity of the method, the results are adequate. We would like to mention that we don't get convergence (or at least very slow convergence) if we don't exlcude particles in the blending domain from the domain of influence across the crack as suggested in section 3.3.

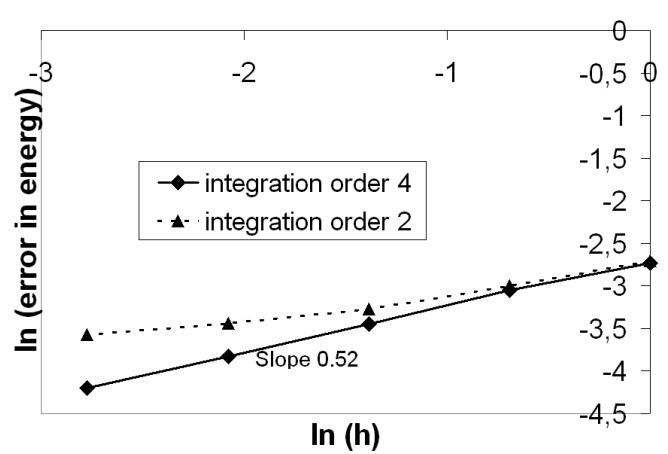

a)

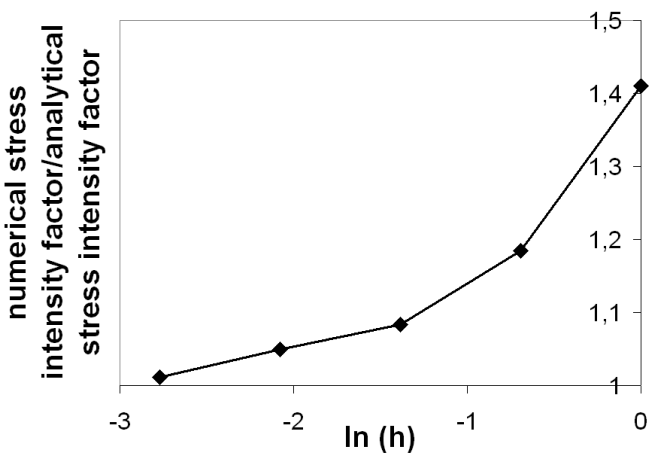

b)

Fig. 7. Convergence for mode I crack problem a) convergence in energy norm (45); b) convergence in $K_{I}$ (integration order is order of Gauss quadrature in cells)

\subsection{Penny crack in a finite tube under uniaxial tension}

Consider a cube of side length $a$ at the center of which a penny-shaped crack of radius $r$ is embedded. The cube is subjected to uniaxial tension $\sigma_{c}=1 \mathrm{mN} / \mathrm{mm}^{2}$. For an infinite plate, the analytical solution for the stress intensity factor is given by $K_{I}=2 \sigma_{c} \sqrt{r / \pi}$. For $a=50 r$, the error in the 


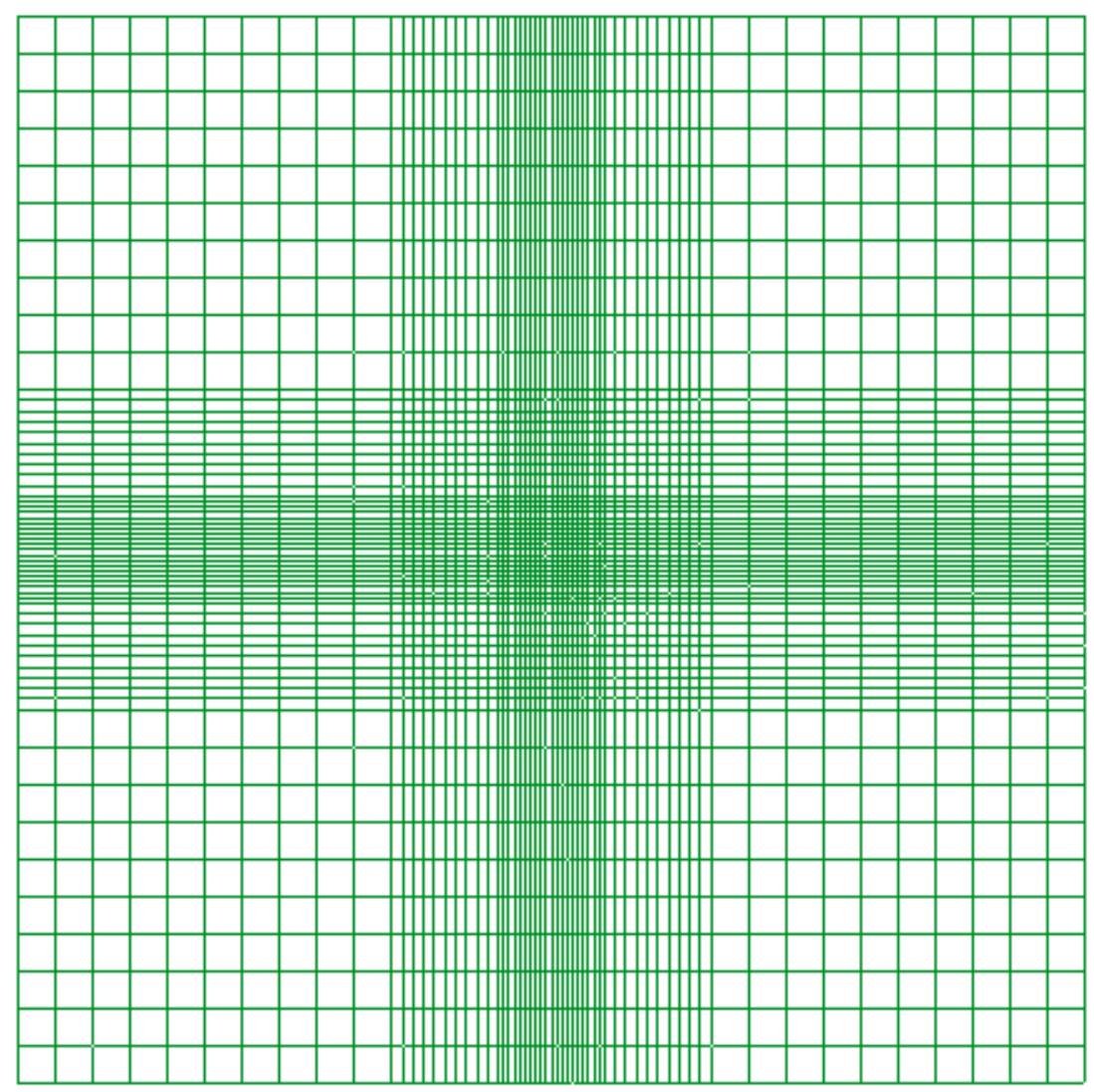

Fig. 8. Typical discretization of the penny crack problem; $2 D$ topview

Table 1

Stress intensity factors for the penny-shaped crack problem

\begin{tabular}{|c|c|c|c|}
\hline Relative error on $K_{I}$ & $30 \times 30 \times 30$ & $60 \times 60 \times 60$ & $120 \times 120 \times 120$ \\
\hline average (\%) & 5.2342 & 3.24 & 2.004 \\
\hline maximum (\%) & 12.635 & 7.74 & 4.33 \\
\hline
\end{tabular}

stress intensity factor is computed for different refinements with different particle spacings $(30 \times 30 \times 30,60 \times 60 \times 60$ and $120 \times 120 \times 120)$; note that the mesh is finer around the crack, see figure 8 in a $2 D$ topview. The average error as well as the maximum error is given in the table 1 . Note that even if no remeshing is necessary, a certain degree of refinement across the crack front is needed to compute accurate stress intensity factors. Adaptivity as proposed in this article is ideally suited to improve the results, but was not used in this problem. 


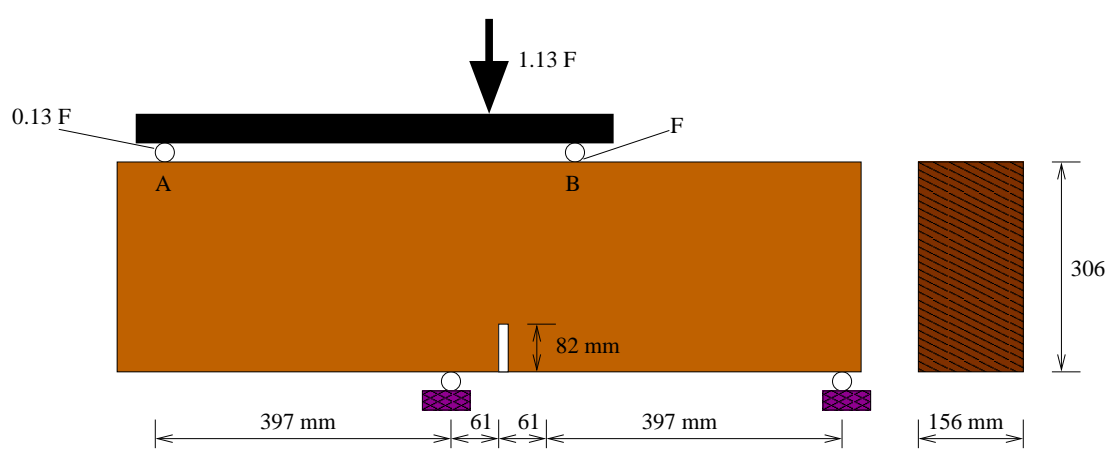

Fig. 9. The tensile/shear beam from Arrea and Ingraffea [2]

\subsection{The notched concrete beam of Arrea and Ingraffea}

The next example is the notched concrete beam of Arrea and Ingraffea [2]. The beam is loaded at points $A$ and $B$, see figure 9 . The initial elastic modulus is 28,000 MPa. The beam fails due to a mixed tensile/shear failure. This problem can be solved quite accurately in 2D. We will use this example to compare our $3 \mathrm{D}$ results with $2 \mathrm{D}$ results obtained in [36] with the same constitutive model.

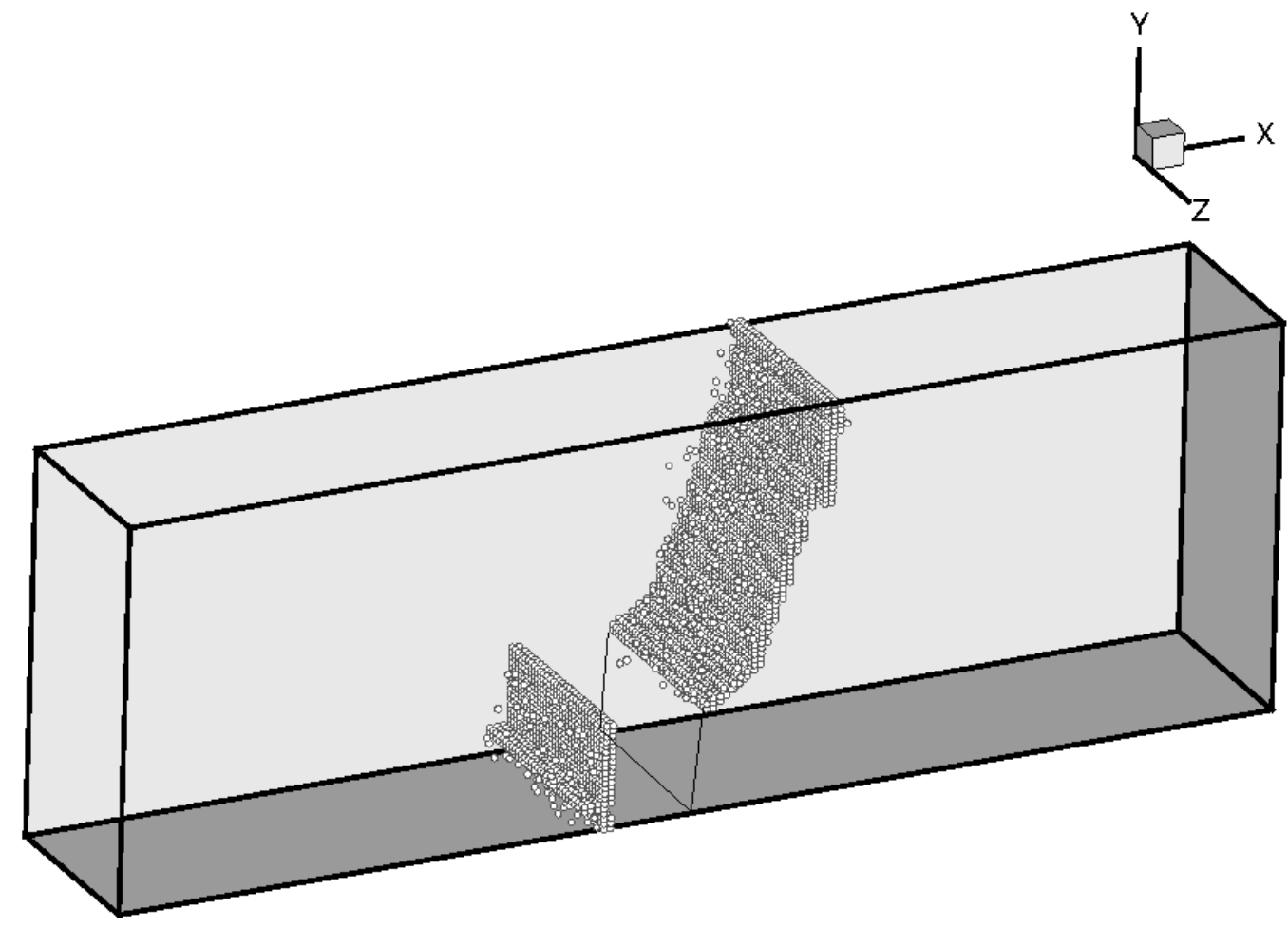

Fig. 10. Crack pattern in the Arrea and Ingraffea [2] beam obtained by the 3D computation using 270,000 particles

We have used the Lemaitre model for the continuum law. Loss of hyperbolicity was used to initiate cracking. For the cohesive traction law, we used a 
linear decay as explained in Belytschko et al. [4]. Two models were used with approximately 270,000 and 2,100,000 particles, respectively.

The $3 \mathrm{D}$ crack pattern for the 270,000 particle simulation at the end of the simulation is shown in figure 10. The curvature of the crack is similar to the one observed in the experiment. Above the left support, a straight crack occurs which also appeared in our 2D computations; this was not observed in the experiment, so evidently our support conditions lead to excessive strain concentrations. Note that some cracked particles appear at a small distance from the main crack; this type of behavior is observed quite often with this method. The load displacement curve (right of the notch) is shown in figure 11 and is similar to the two dimensional one.

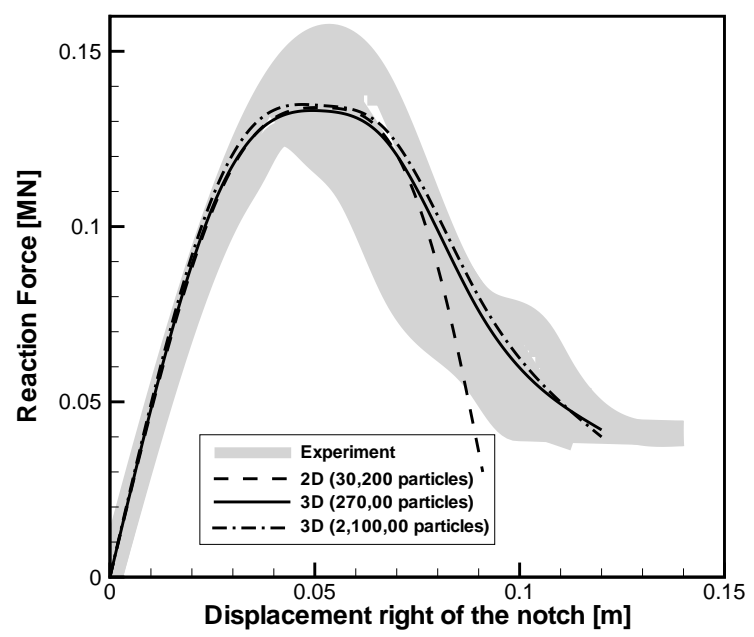

Fig. 11. Load-displacement curve (to the right of the notch) of the Arrea and Ingraffea [2] beam; experimental results are compared to a $2 \mathrm{D}$ and to $3 \mathrm{D}$ computations

\section{$5.4 \quad$ Non-planar crack growth}

A more complicated example involves non-planar crack growth. This example was studied with the extended finite element method by Gravouil et al. [22] A beam is notched at an angle $\alpha$ of 45 degrees with the x-axis (see figure 12) and loaded in bending by a moment at the ends as shown in figure 12 . The beam dimensions are $h=0.02 \mathrm{~m}, l=0.1 \mathrm{~m}$ and $d=0.01 \mathrm{~m}$ and the length of the notch is $a=0.01 \mathrm{~m}$.

In Gravouil et al. [22], the crack surface tended to become normal to the xaxis as it grew downwards. Computations with up to approximately 170,000 particles did not give acceptable results; the crack did not curve and went straight down. For a slightly higher numbers of particles, the crack curved 


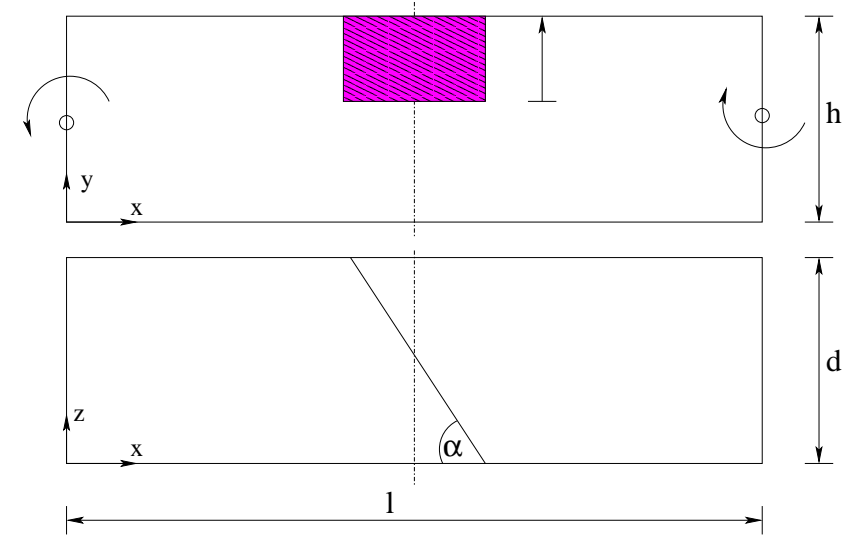

Fig. 12. Side and top views of the beam with an initial crack for the non-planar crack growth

slightly but did not reach the correct final position, which is perpendicular to the x-axis. With 570,000 particles, the results become independent of the discretization and the end of the crack is approximately $0.002 \mathrm{~m}$ above the bottom of the beam. The crack for 570,000 $(301 \times 31 \times 61)$ and 4,400,000 $(601 \times 61 \times 121)$ particles (and 540,000 and 4,300,000 stress points, respectively) is shown in figure 13 and is in good agreement with the results obtained in Gravouil et al. [22].

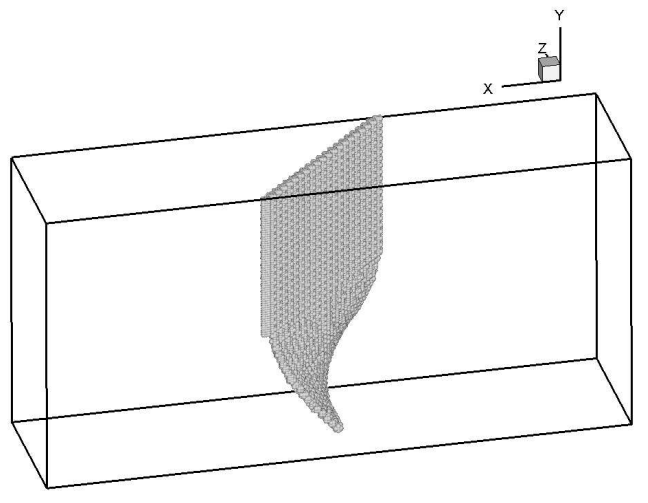

a)

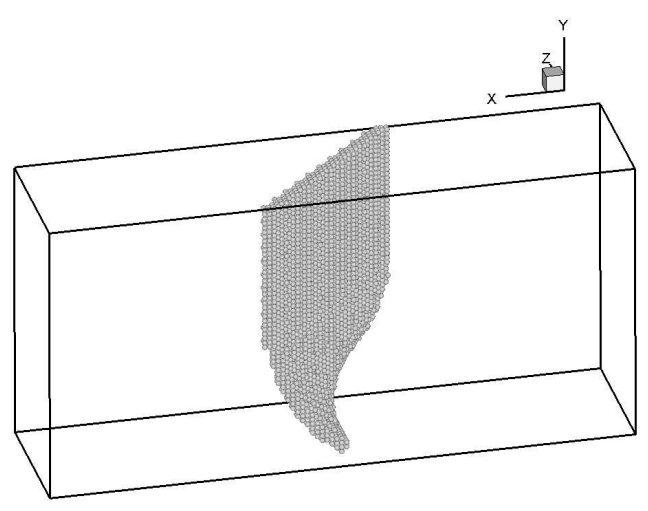

b)

Fig. 13. Non-planar crack for a) 4,400,000 particles and b) 570,000 particles; a cutout near the crack is shown

We applied also our adaptive scheme to this problem, starting with discretizations of 23,000 particles $(101 \times 11 \times 21)$ and 75,000 particles $(151 \times 16 \times 31)$. The cracks for these two computations are presented in figure 14 and are almost identical and also to the results obtained with the 570,000 particle simulation without adaptivity. At the end of the simulation, the number of particles was around 65,000 and 210,000 particles for the 23,000 and 75,000 adaptive particle discretizations, respectively. The adaptive computations were around ten times faster. 


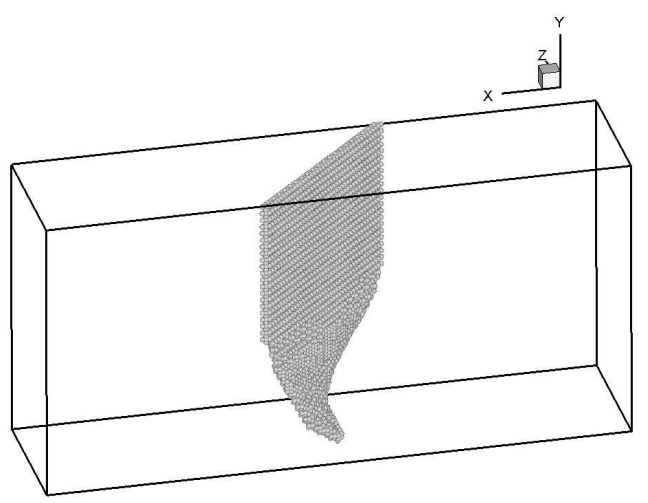

a)

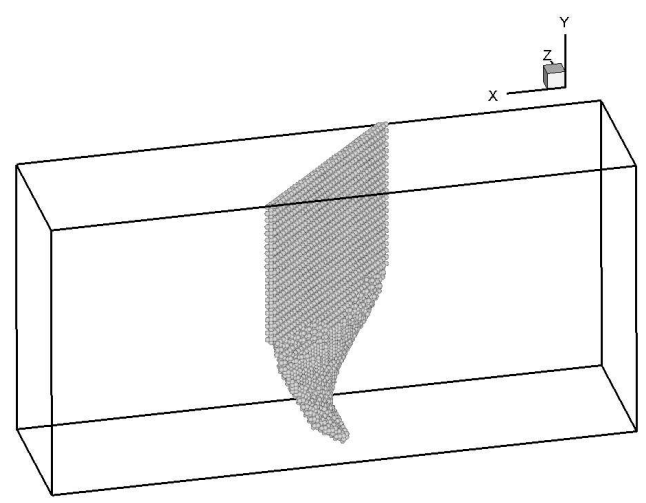

b)

Fig. 14. Crack pattern of the adaptive method for an initial particle number of c) 23,000 particles and d) 75,000 particles; a cutout near the crack is shown

\subsection{Failure of a reinforced concrete beam}

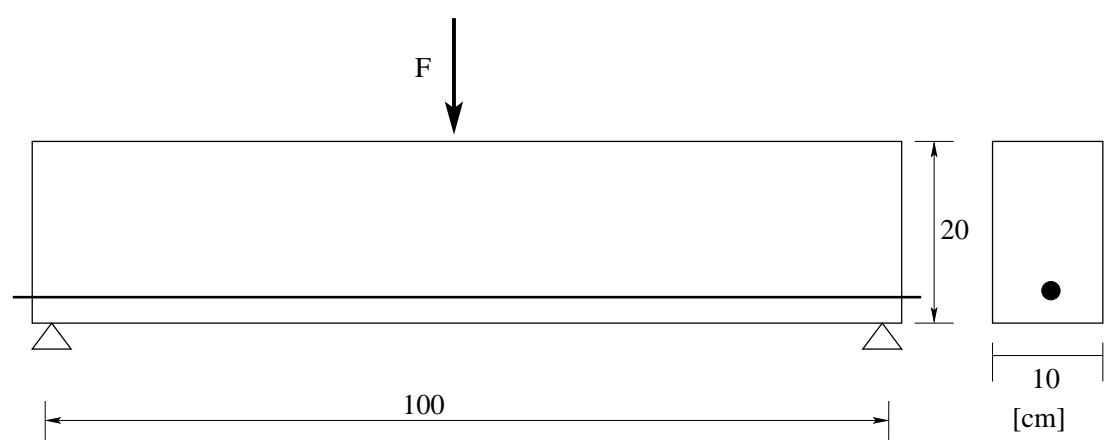

Fig. 15. Reinforced concrete beam, model description

Consider a reinforced concrete beam in three-point-bending as shown in figure 15. The concrete is reinforced with one bar at the bottom and the beam is of rectangular cross section. We consider bond effects between the concrete and the reinforcement with the rheological model illustrated in figure 16. Typical bond curves depending on the confinement (tensile) stress are shown in figure 17. The wider the crack opens, the smaller are the bond stresses. A more detailed description of the bond model is given in Rabczuk and Belytschko [35].

We employed the Rankine criterion in tension and linear elastic material behavior in compression. Young's modulus for concrete is 36,000 MPa, Poisson's ratio is 0.22 and the cutoff stress is 3.0 MPa. Beam elements are used for the discretization of the reinforcement. An elasto-plastic model with isotropic hardening is used for the steel. Young's modulus of the reinforcement is 210,000 $M P a$, Poisson's ratio is 0.3 and the yield stress is $510 \mathrm{MPa}$. 


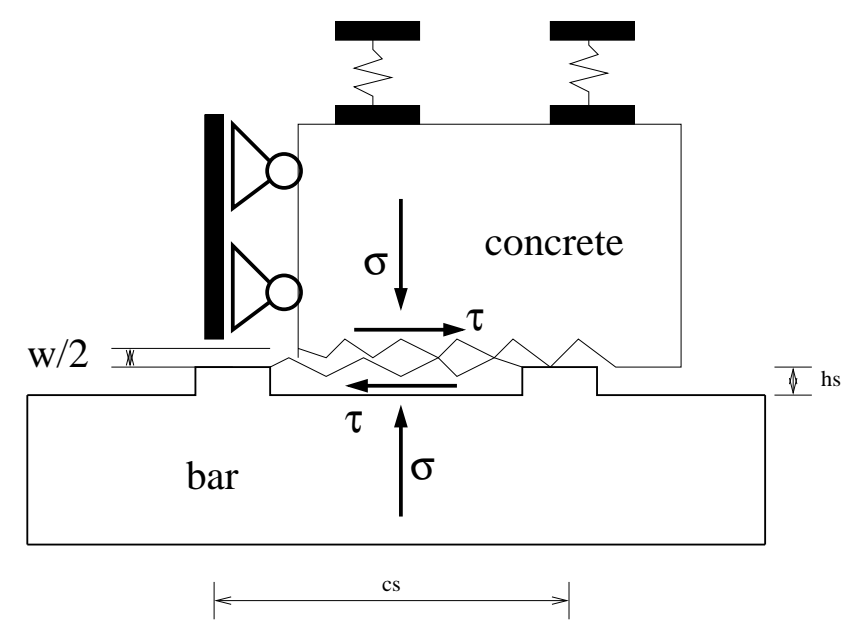

Fig. 16. Rheological model of the bond approach

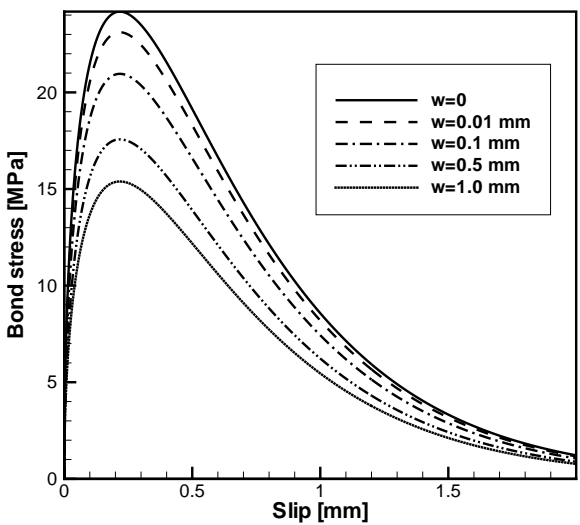

a)

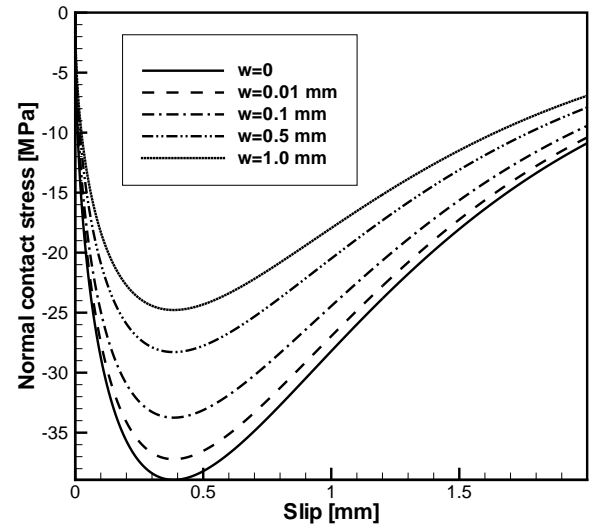

b)

Fig. 17. a) Bond stress slip curve depending on the crack width $w$, b) Normal (contact) stress slip curve

The crack pattern at failure is shown in figure 18. No experimental data is available but the crack pattern looks reasonable. The cracks closer to the supports are flatter and become steeper with decreasing distance to the applied load. The cracks appear at a characteristic spacing that is reproduced very well independent of refinement after a certain point. At the end of the computation the concrete around the bar is completed cracked. The load displacement curve is shown in figure 19 for two different particle discretizations. A sudden drop in the load displacement curve can be observed at a displacement of $0.5 \mathrm{~cm}$. The beam fails in bending. 


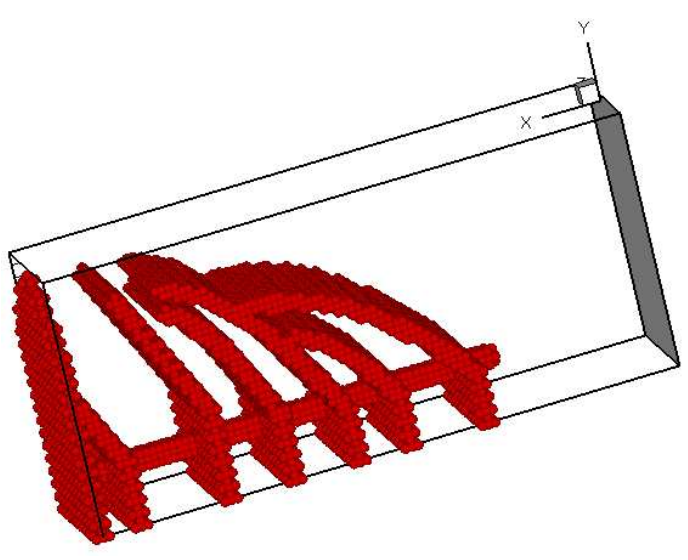

a)

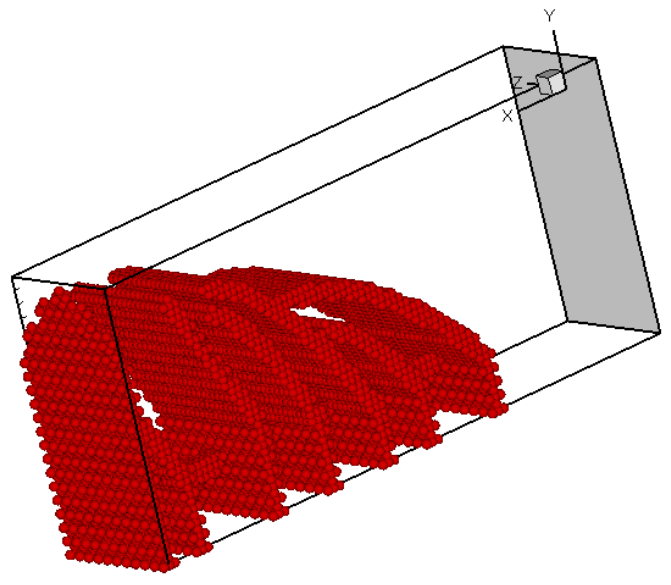

b)

Fig. 18. Crack pattern of the reinforced concrete beam at failure from two different viewing points

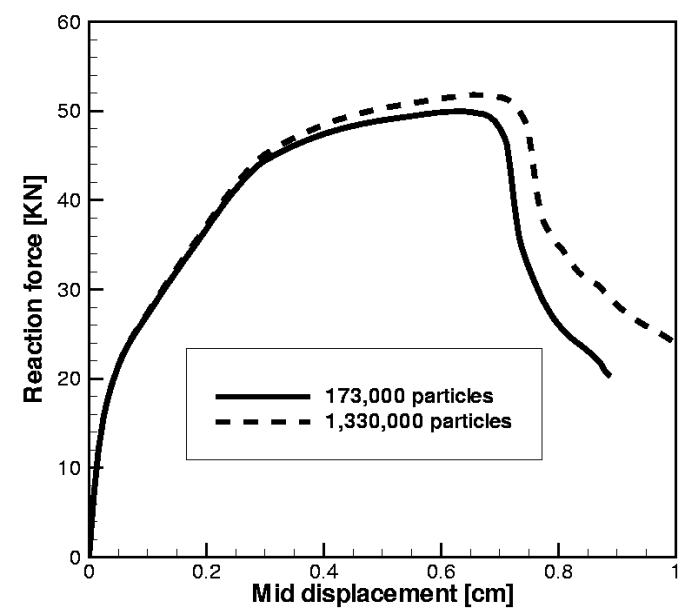

Fig. 19. Load deflection curve of the three-point-reinforced concrete beam

\subsection{Crack Branching}

Consider a rectangular prenotched specimen as shown in figure 20. The length of the specimen is $0.1 \mathrm{~m}$, width $0.04 \mathrm{~m}$ and thickness $0.004 \mathrm{~m}$. Tensile tractions of $1 \mathrm{MPa}$ are applied on the top and bottom edges, see figure 20. This example was recently studied by Rabczuk and Belytschko [36] in 2D under plane strain assumptions. Computations in 2D have also been reported by $\mathrm{Xu}$ and Needleman [47] and Falk et al. [21] using interelement methods and Belytschko et al. [4] using the extended finite element method. We use here the Lemaitre damage model [26] and loss of hyperbolicity to transition to a discontinuity. The material parameters can be found in Rabczuk and Belytschko [36].

We will show results for a 368,000 particle model. Similar results were obtained 


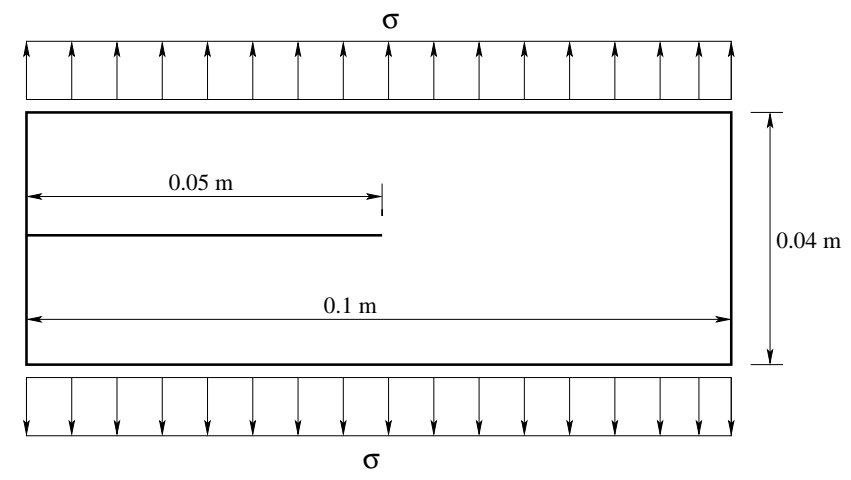

Fig. 20. Plate with an edge crack

Table 2

Material parameters of the constitutive model for concrete

\begin{tabular}{|c|c|c|}
\hline$E_{0}=58,000 M P a$ & $e_{0}=1.0 \cdot 10^{-4}$ & $e_{p 2}=0.4$ \\
\hline$\nu=0.22$ & $e_{d}=3.0 \cdot 10^{-4}$ & $\varrho=2.77 \cdot 10^{-3}$ \\
\hline$c_{1}=0.0123424$ & $g_{d}=2.2$ & $n_{d a m}=70$ \\
\hline$c_{2}=0.025166$ & $r_{t}=1.2$ & $a_{v}=0.7$ \\
\hline$c_{3}=0.782058$ & $r_{c}=20$ & $b_{v}=3.5$ \\
\hline$c_{4}=0.346384$ & $c_{p}=0.9$ & $e_{v}=0.02$ \\
\hline$c_{c}=0.08$ & $e_{p 1}=1.1 \cdot 10^{-3}$ & $e_{v, t h}=0.008$ \\
\hline
\end{tabular}

with finer refinements. In addition, we have carried out computations with adaptivity.

The crack pattern at different time steps for the 368,000 particle arrangement is shown in figure $21 \mathrm{a}-\mathrm{c}$. In figure $21 \mathrm{~d}-\mathrm{f}$, the crack pattern for an adaptive simulation is shown. We started with approximately 40,000 particles and set an upper limit of 400,000 particles. The crack pattern is smoother than for the 368,000 particle non-adaptive computation. We also ran adaptive computations with different upper bounds for the number of particles and different initial numbers of particles (about 40,000 particles). The results were very similar and the crack paths were quite smooth for these calculations.

A good measure for the quality of such computations is the energy balance check. For this problem, the error in the energy balance is less than $1 \%$.

\subsection{Concrete impact}

Unosson and Nilsson [45] performed a series of impact experiments on cylindrical concrete and reinforced concrete slabs. In the experiments, a steel projectile of $6.3 \mathrm{~kg}$ impacted cylindrical reinforced concrete structures of different 


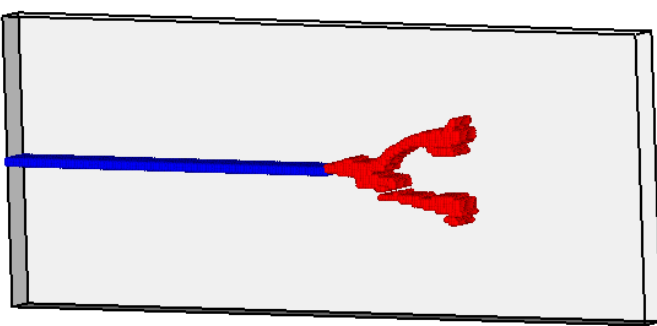

a) $0.03 \mathrm{~ms}$

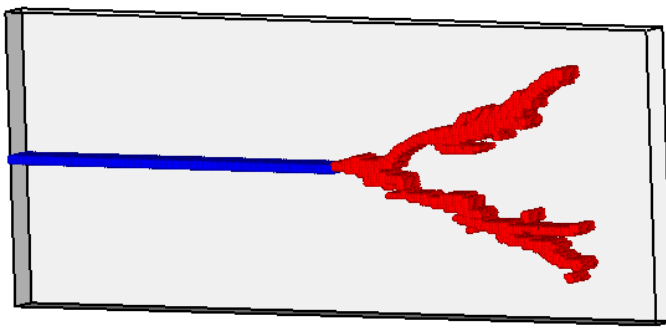

b) $0.046 \mathrm{~ms}$

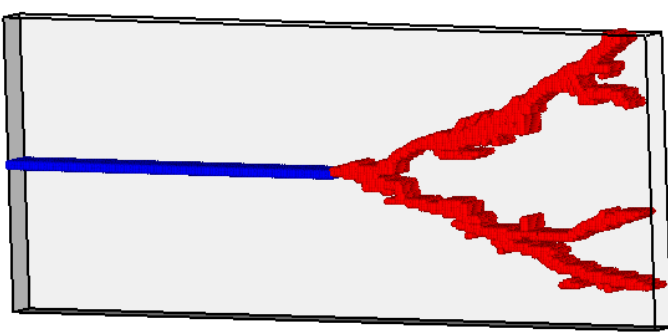

c) $0.06 \mathrm{~ms}$

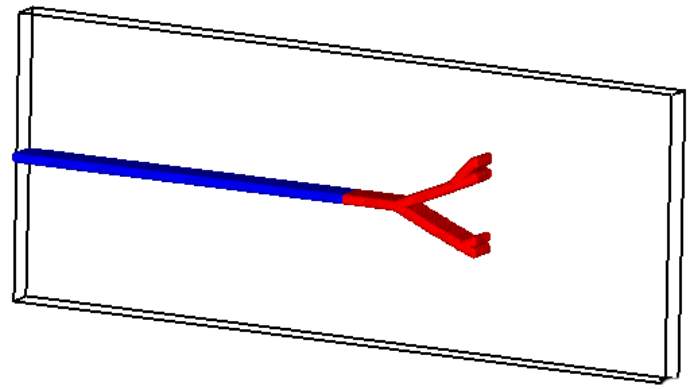

d) $0.03 \mathrm{~ms}$

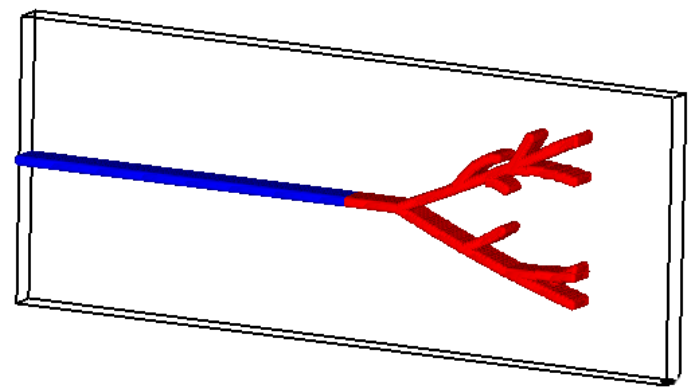

e) $0.046 \mathrm{~ms}$

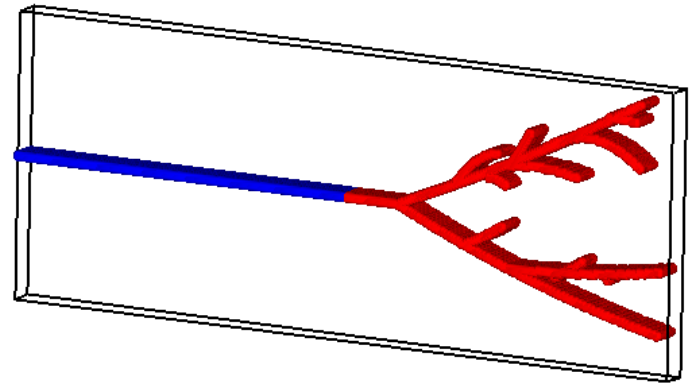

f) $0.06 \mathrm{~ms}$

Fig. 21. Three-dimensional crack branching at different time steps, a)-c) non-adaptive, d)-f) adaptive

thicknesses. The total length of the projectile is $225 \mathrm{~mm}$ and its diameter 75 $\mathrm{mm}$. The ogival nose radius of the projectile is $127 \mathrm{~mm}$. We consider a series of impacts on two concrete targets. The first concrete slab has a diameter of $1.4 \mathrm{~m}$ and a thickness of $0.4 \mathrm{~m}$. The second concrete slab has also a diameter of $1.4 \mathrm{~m}$ but the thickness is $0.8 \mathrm{~m}$.

Unosson and Nilsson [45] measured the residual velocity of the projectile. For the $0.4 m$ thick concrete slab, perforation of the concrete specimen was observed while for the structures of $0.8 m$ thickness, the projectile was stopped in the middle of the concrete slab. The experimental results are shown in table 3 .

We discretized the concrete structures with a structured particle arrangement. The constitutive model described in section 4.1.2 is employed. For the steel 
Table 3

Impact experiments on concrete slabs by Unosson and Nilsson [45]

\begin{tabular}{|c|c|c|c|}
\hline Target & Impact Velocity $[\mathrm{m} / \mathrm{s}]$ & Penetration depth $[\mathrm{m}]$ & Residual projectile velocity $[\mathrm{m} / \mathrm{s}]$ \\
\hline $1: 1$ & 613 & - & 276 \\
$1: 2$ & 616 & - & 303 \\
$1: 3$ & 621 & - & 293 \\
$2: 1$ & 623 & 0.45 & - \\
$2: 2$ & 618 & 0.54 & - \\
$2: 3$ & 622 & 0.51 & - \\
\hline
\end{tabular}

projectile, an elasto-plastic material law with isotropic hardening is applied. In the experiments, the deformation of the projectiles was small as confirmed by the simulations. The material parameters for the concrete are given in table 2. Loss of material stability is used for crack initiation. For the steel projectile, Young's modulus is 210,000 $\mathrm{MPa}$, Poisson's ratio 0.3 , density $0.008 \mathrm{~g} / \mathrm{mm}^{3}$, yield strength $2400 \mathrm{MPa}$ and the hardening modulus is 20,000 $\mathrm{MPa}$.

We will focus first on experiments 1:1 to 1:3. We used two different refinements, 630,000 particles (and 595,000 stress points) and approximately 5, 200,000 particles (and 5,050,000 stress points). A plot of the impactor penetrating the concrete slab at the beginning of the computation is shown in figure 22a. The deformed concrete slab at different times and for different refinements is shown in figure 22a to d. The results do not exhibit much mesh dependence. The final deformation of the concrete slab is shown in figure 23 and matches quite well with the experimental crack pattern (see Unosson and Nilsson [45]) though the crater diameter is slightly underestimated by the numerical computation; however the scatter in such impact experiments can be quite large. The residual velocity of the projectile can be reproduced by the numerical computation quite well and is independent of the discretization for the ones considered. The impactor perforates the concrete slab at approximately $0.5 \mathrm{~ms}$ after first contact with the concrete slab which matches well the experimental perforation time measured in [45]. The numerical results are summarized in table 4 .

The error of the energy in time for this problem is shown in figure 24 for two discretizations and does not exceed $10 \%$. For the finer discretization the error in the energy is $5 \%$. For the penetration problem (experiment 2:1 to 2:3) that is discussed next, the error in the energy is also around $5 \%$.

Experiments 2:1 to 2:3 are also discretized with different refinements. We will show the results for our finest discretization with more than 9.800,000 particles. The other results are similar. The material parameters for the concrete slab and the steel projectile are the same as before and can be found above 


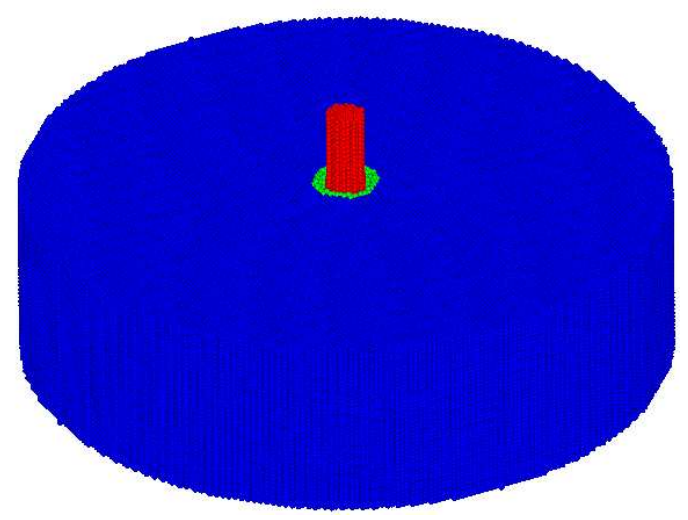

a) $0.1 \mathrm{~ms}$

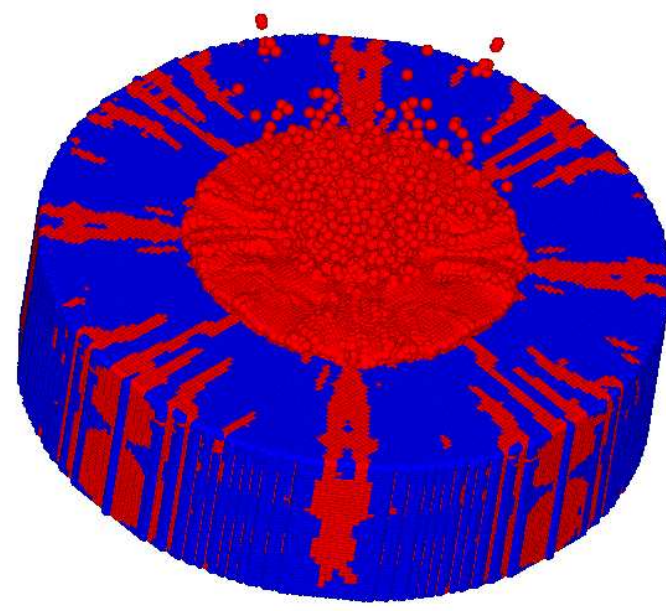

c) $3.5 \mathrm{~ms}$

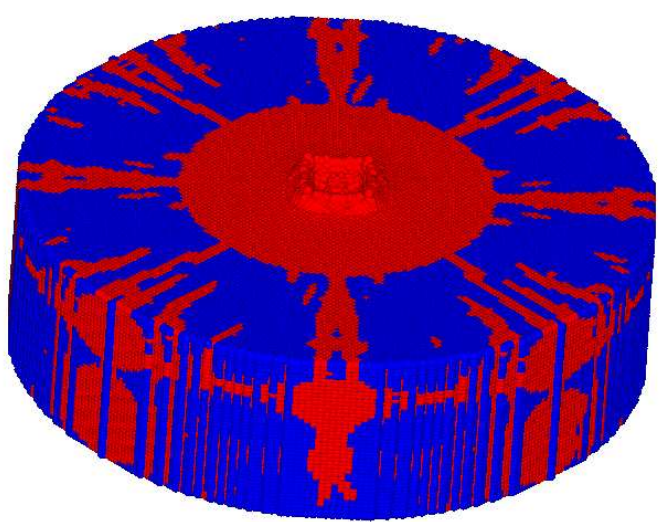

b) $0.9 \mathrm{~ms}$

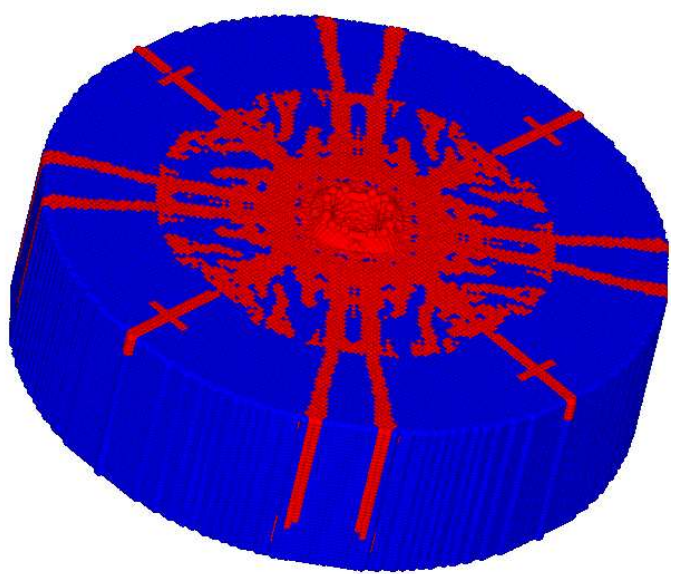

d) $0.55 \mathrm{~ms}$

Fig. 22. Simulation of experiment 1:1 to 1:3; a), b) and c) for the 630,000 particle discretization and d) for the 5,200,000 particle discretization

Table 4

Numerical results of the impact experiments on concrete slabs by Unosson and Nilsson [45]

\begin{tabular}{|c|c|c|c|c|}
\hline $\begin{array}{c}\text { Number of } \\
\text { particles }\end{array}$ & Target & $\begin{array}{c}\text { Impact Velocity } \\
{[\mathrm{m} / \mathrm{s}]}\end{array}$ & $\begin{array}{c}\text { Penetration depth } \\
{[\mathrm{m}]}\end{array}$ & $\begin{array}{c}\text { Residual projectile } \\
\text { velocity }[\mathrm{m} / \mathrm{s}]\end{array}$ \\
\hline 630,000 & $1: 1$ to $1: 3$ & 616 & - & 278.0 \\
$5,200,000$ & $1: 1$ to $1: 3$ & 616 & - & 283.0 \\
$9,800,000$ & $2: 1$ to $2: 3$ & 617 & 0.55 & - \\
\hline
\end{tabular}

and in table 2 .

Figure 25a shows the crack pattern of the concrete structure at $0.8 \mathrm{~ms}$. Only every second particle is plotted. It is similar to the crack pattern of the thin slab but crack branching at the top surface occurs early in the simulation and the crater dimensions are smaller. Also the round shape of the crater obtained 


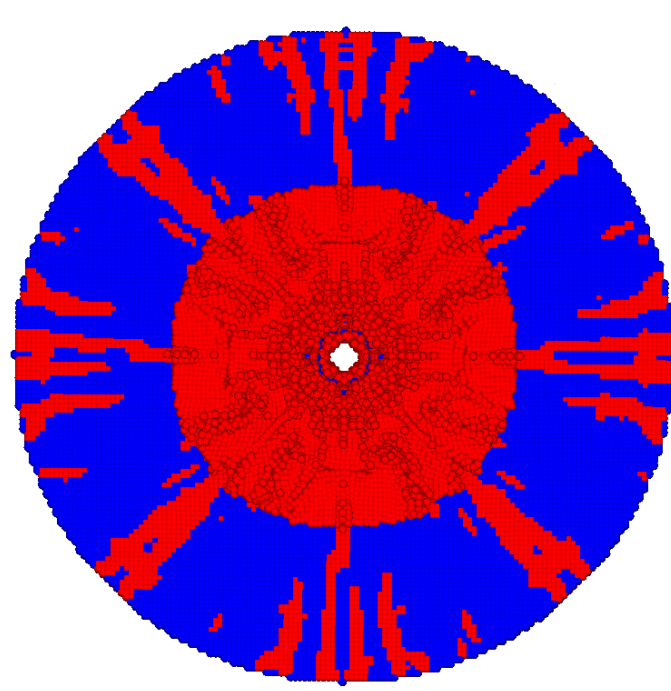

a) simulation

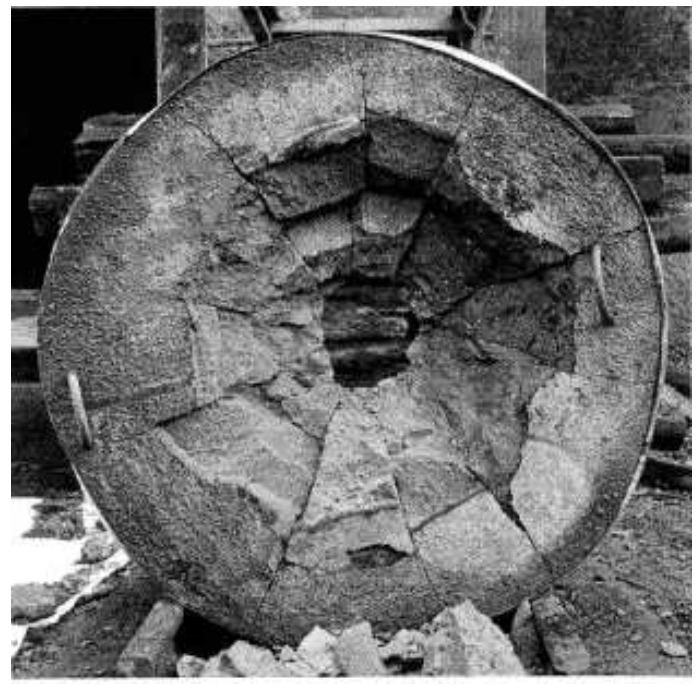

b) experiment

Fig. 23. Crack pattern of the simulation (630,000 particles) compared to the experimental crack pattern by Unosson and Nilsson [45]; experiment 1:1 to 1:3

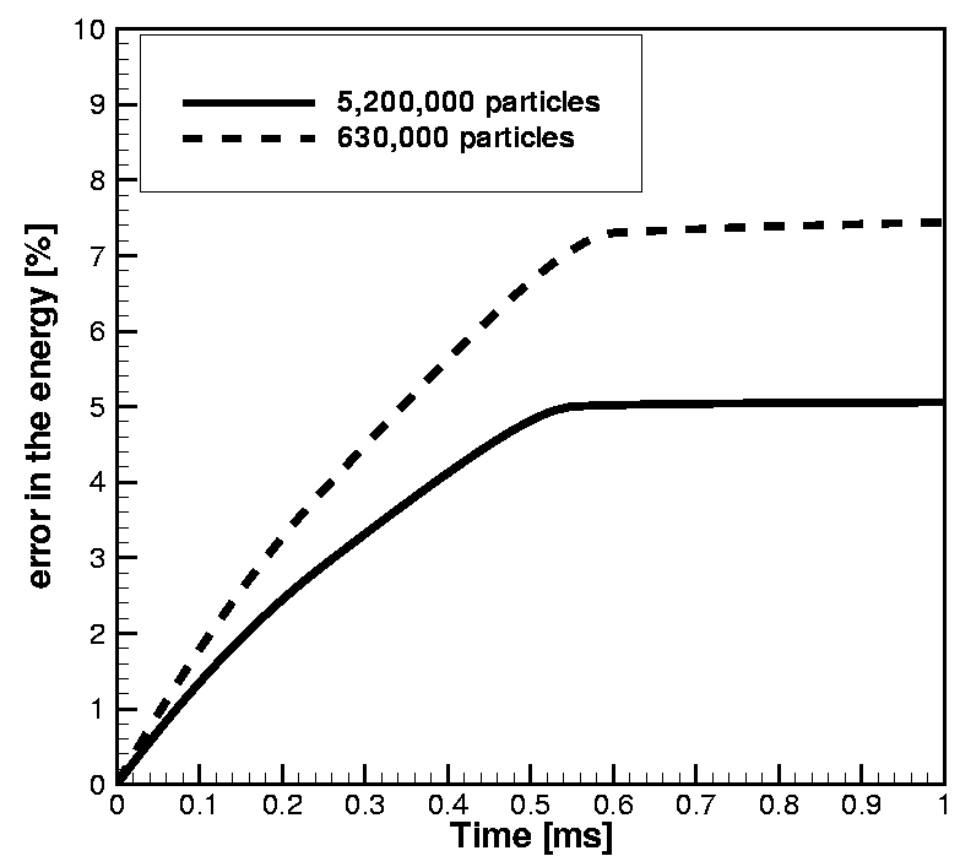

Fig. 24. Error in the energy for the perforated Unosson impact problem for the experiment 1:1 to 1:3 changed.

Figure 25b shows the crack pattern through a cross-section of the structure. It can be seen that the penetration depth is approximately $0.55 \mathrm{~m}$ which matches well with the experimental results. A high damage zone around the projectile can be observed. The damage of the slab is larger at the top and of the bottom of the structure. Spalling at the bottom can be identified. The middle of the structure is less damaged. Note that a cut in another plane of the structure 
could look quite different (compare to the crack pattern of the full structure, figure 25a). We carried out this computation until approximately $0.8 \mathrm{~ms}$. This is not sufficient to study the fragmentation or spalling of the structure but it is sufficient to capture the penetration depth. The projectile reaches its final position at around $0.55 \mathrm{~ms}$.

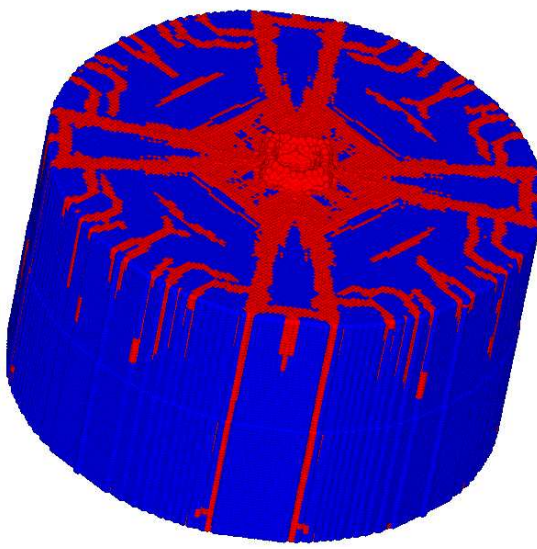

a) simulation

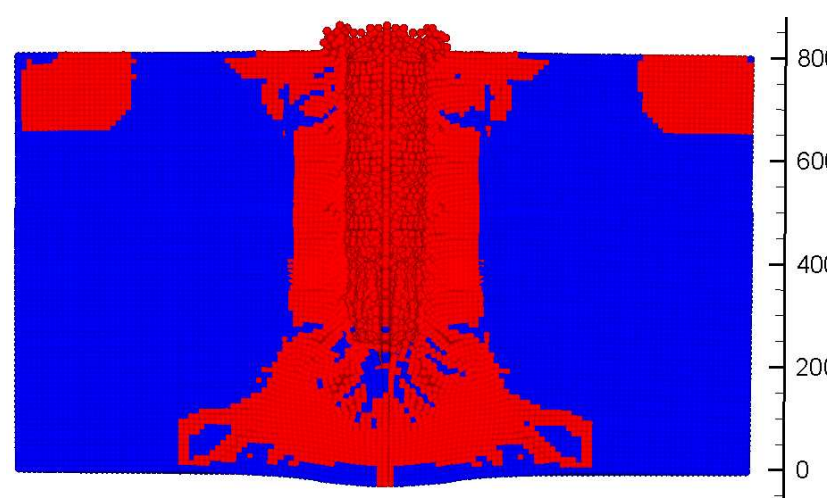

b) cross-section

Fig. 25. Crack pattern of the simulation of experiments 2:1 to 2:3, a) surface view, b) cross section

\subsection{Concrete under contact detonation}

These computations are compared with tests of concrete slabs with different thicknesses and strengths performed by Hermann [23] and Ockert [32]. The numerical results are compared with the data from two experiments (slab O1 and H1). The schematic of the test setup is illustrated in figure 26a; a typical discretization of the slab is shown in figure 26b. The yellow cone shows the explosive and the concrete slab is in blue.

The concrete slab $O 1$ has a thickness of $25 \mathrm{~cm}$ and an area of $100 \mathrm{~cm} \times 100 \mathrm{~cm}$. The compressive strength of the concrete is $48 \mathrm{MPa}$. The slab $H 1$ has a thickness of $50 \mathrm{~cm}$ and an area of $100 \mathrm{~cm} \times 100 \mathrm{~cm}$ and the compressive strength is also $48 \mathrm{MPa}$. The two slabs were loaded with a plane wave generator which consists of an outer cone of Composition B and an inner cone of TNT. The cone has a diameter of $10.3 \mathrm{~cm}$ and its height is $7.5 \mathrm{~cm}$ for both experiments. At the middle of the slab $H 1$ directly under the plane wave generator, hydrostatic pressures were measured. The measured pressures are compared with those obtained by our simulations. Slab $O 1$ was completely perforated in the experiment while slab $H 1$ was only penetrated because of its larger thickness.

We tried different numbers of particles for the simulations. For slab $H 1$, we used between 380,000 and 4,000,000 particles. We will show the results for these two cases. Calculations with intermediate numbers of particles gave 


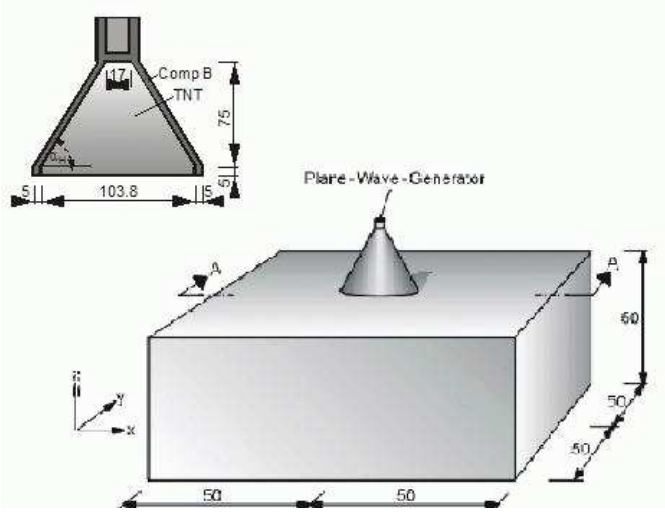

a)

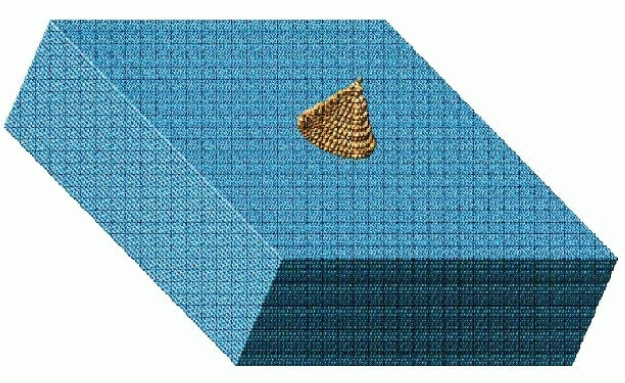

b)

Fig. 26. a) Schematic of the test set up of the explosive experiments, b) Discretization of the concrete slab

Table 5

Material parameters of the constitutive model for concrete

\begin{tabular}{|c|c|c|}
\hline$E_{0}=36,000 \mathrm{MPa}$ & $e_{0}=2.5 \cdot 10^{-5}$ & $e_{p 2}=0.4$ \\
\hline$\nu=0.22$ & $e_{d}=2.2 \cdot 10^{-4}$ & $\varrho=2.4 \cdot 10^{-3}$ \\
\hline$c_{1}=0.0123424$ & $g_{d}=1.5$ & $n_{d a m}=70$ \\
\hline$c_{2}=0.025166$ & $r_{t}=1.2$ & $a_{v}=0.7$ \\
\hline$c_{3}=0.782058$ & $r_{c}=20$ & $b_{v}=3.5$ \\
\hline$c_{4}=0.346384$ & $c_{p}=0.9$ & $e_{v}=0.02$ \\
\hline$c_{c}=0.08$ & $e_{p 1}=1.1 \cdot 10^{-3}$ & $e_{v, t h}=0.008$ \\
\hline
\end{tabular}

Table 6

Material parameters of the explosive

\begin{tabular}{|c||c|c|c|c|c|c|c|c|c|c|}
\hline & $\begin{array}{c}\varrho_{0} \\
{\left[\mathrm{~g} / \mathrm{mm}^{3}\right]}\end{array}$ & $\begin{array}{c}P_{\max } \\
{[M P a]}\end{array}$ & $\begin{array}{c}c_{D} \\
{[\mathrm{~m} / \mathrm{s}]}\end{array}$ & $\begin{array}{c}E_{m 0} \\
{[M P a]}\end{array}$ & $\begin{array}{c}A \\
{[M P a]}\end{array}$ & $\begin{array}{c}B \\
{[M P a]}\end{array}$ & $\begin{array}{c}C \\
{[M P a]}\end{array}$ & $R_{1}$ & $R_{2}$ & $\omega$ \\
\hline TNT & 0.00163 & 21,000 & 6930 & 70,000 & 37,120 & 3231 & 1045 & 4.15 & 0.95 & 0.3 \\
\hline Comp. B & 0.001717 & 29,500 & 7980 & 85,000 & 52,420 & 7678 & 1082 & 4.2 & 1.1 & 0.34 \\
\hline
\end{tabular}

similar results. For slab $O 1$, we used approximately 265,000 and 2,120,000 particles. The viscous damage model and the corresponding cohesive model as explained in section 4.1.2 are used. Loss of material stability is used for crack initiation. The parameters for the constitutive law are listed in table 5 . The explosive is simulated by a John-Wilkonson-Lee (JWL) equation of state (EOS):

$$
p=A\left(1-\frac{\omega \varrho}{R_{1} \varrho_{0}}\right)^{-R_{1} \frac{\varrho_{0}}{\varrho}}+B\left(1-\frac{\omega \varrho}{R_{2} \varrho_{0}}\right)^{-R_{2} \frac{\varrho_{0}}{\varrho}}+\frac{\omega \varrho^{2}}{\varrho_{0}} E_{m 0}
$$


The material parameters for the EOS are listed in table 6 .

The deformation of the concrete slab $O 1$ and the explosive at the beginning of the computation are shown in figure 27. Extremely large deformation, especially for the explosive can be observed. An Eulerian kernel is used to simulate the explosive.

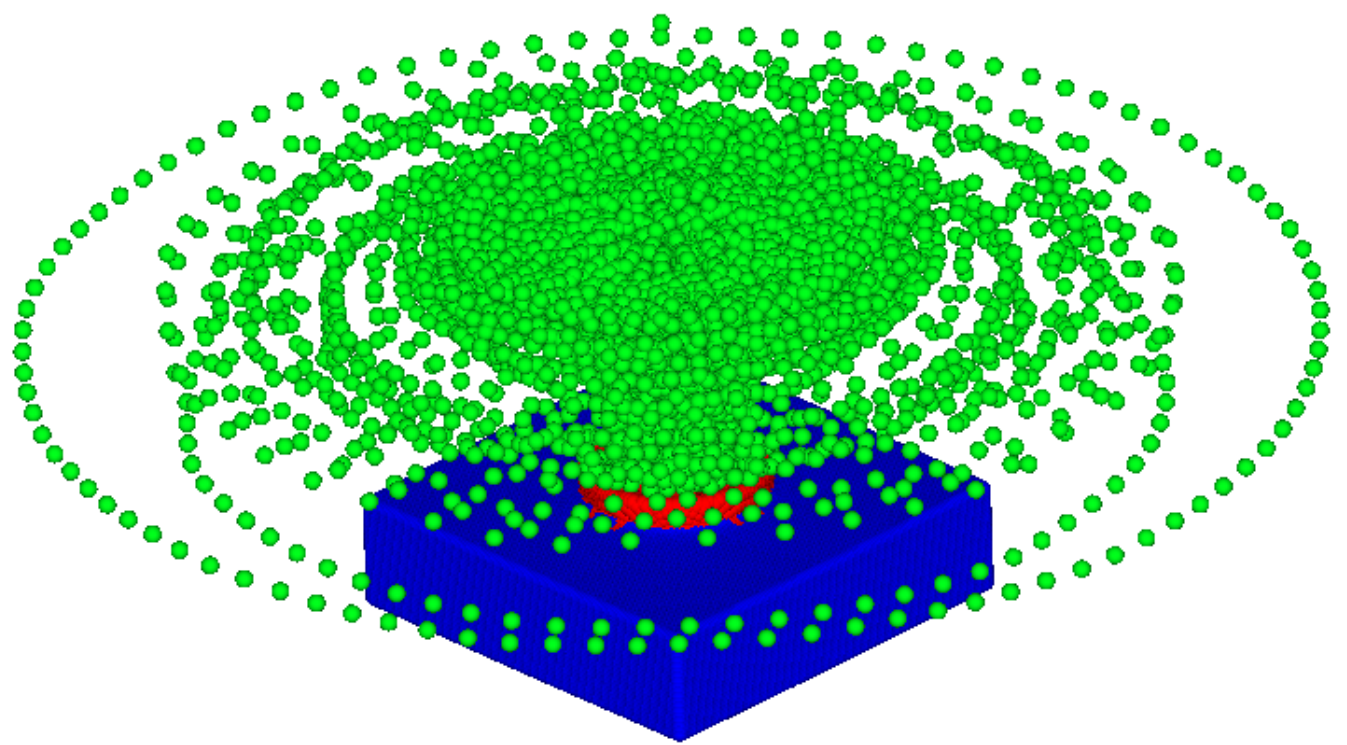

Fig. 27. Concrete slab $O 1$ with explosive near the beginning of the calculation; in red: cracked particles, in blue: uncracked particles, in green: explosive

Figure 28 shows a bottom and top point of view at different time steps of the concrete slab $O 1$ using 265,000 particles. As can be seen, the cracking process is completed at $0.5 \mathrm{~ms}$. Afterwards, the slab only fragments. Multiple cracks and crack branching can be observed. The latter occurs when the cracks approach the edge of the slab. It should be mentioned that 265,000 particle is a rather coarse discretization for a 3D simulation. In the vertical plane, we have only 2,626 particles while in the horizontal projection we have 10,201 particles. In the $2 \mathrm{D}$ crack branching problem described in [36], approximately 12,000 particles were necessary to obtain acceptable results in the crack patterns.

The crack pattern and deformation for the 2,120,000 particle simulation is shown in figure 29 and is very similar to the crack pattern with the coarse discretization. More cracks can be observed which propagate radially from the crater. Also more crack branching occurs especially at the bottom of the structure. The time at which cracking is completed coincides well with the results from the coarse simulation $(0.5 \mathrm{~ms})$. We performed the computation up to $5 \mathrm{~ms}$. The deformed concrete structure at $2.5 \mathrm{~ms}$ is shown in figure 30 . No instabilities are observed. Some cracked particles which formed the crack at the bottom side peeled away from the structure. 


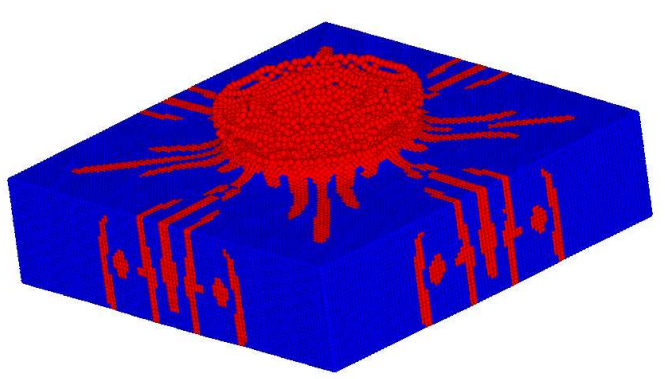

(a) $0.2 \mathrm{~ms}$

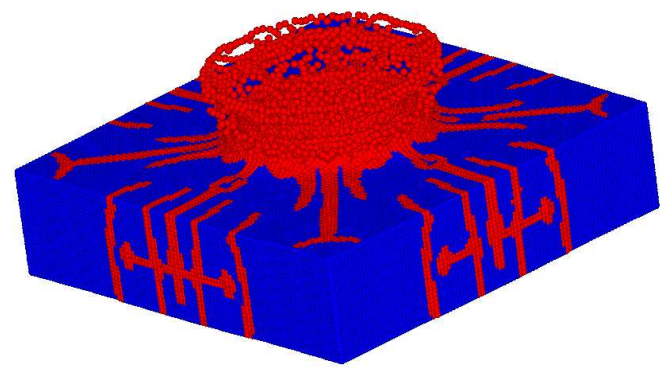

(c) $0.4 \mathrm{~ms}$

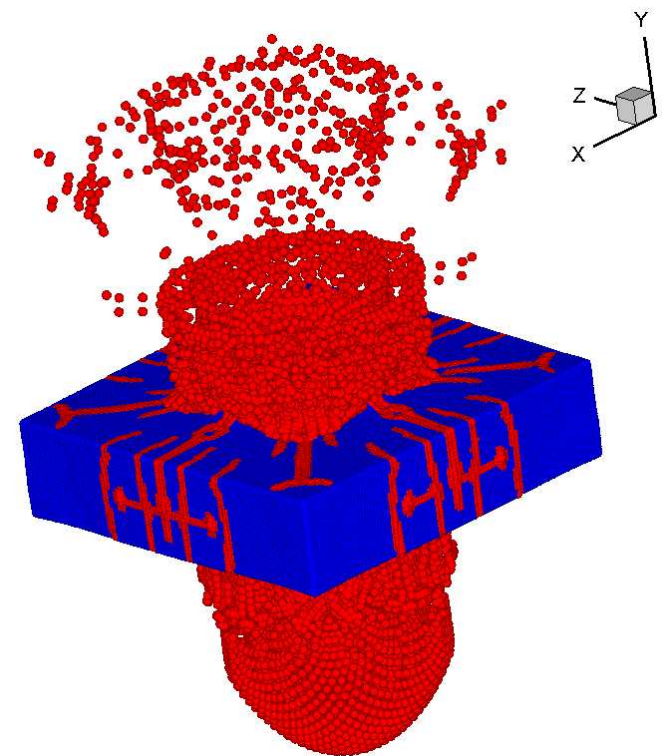

(e) $1.65 \mathrm{~ms}$

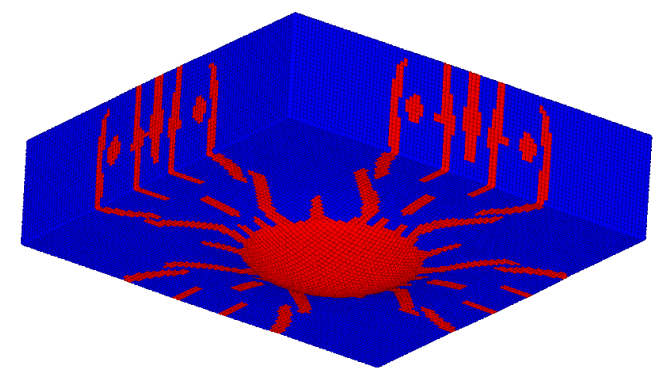

(b) $0.2 \mathrm{~ms}$

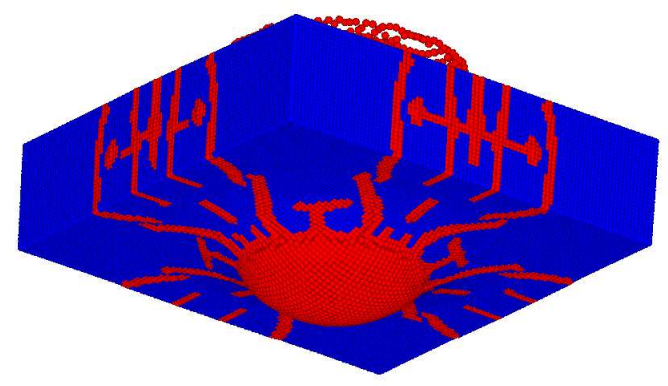

(d) $0.4 \mathrm{~ms}$

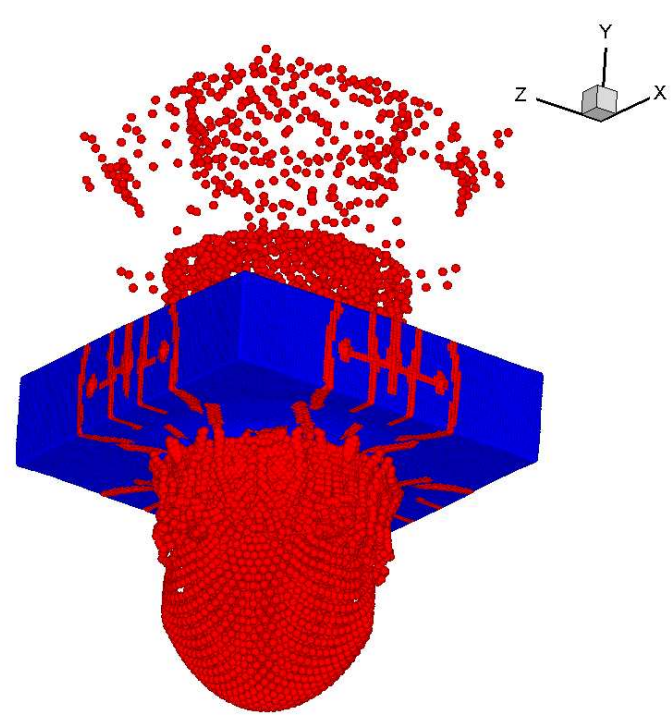

(f) $1.65 \mathrm{~ms}$

Fig. 28. Concrete slab O1 under explosive loading using approximately 265,000 particles, in red: cracked particles, in blue: uncracked particles

The crater dimensions are approximately the same in both simulations. The perforation is reproduced well in both computations. A picture of the experimentally observed slab $O 1$ is shown in figure 32 and agrees very well with the crack pattern and the perforation crater of the simulation. Note that only every second particle is shown in the deformation plots in figure 29 due to postprocessing problems if all particles are shown. 


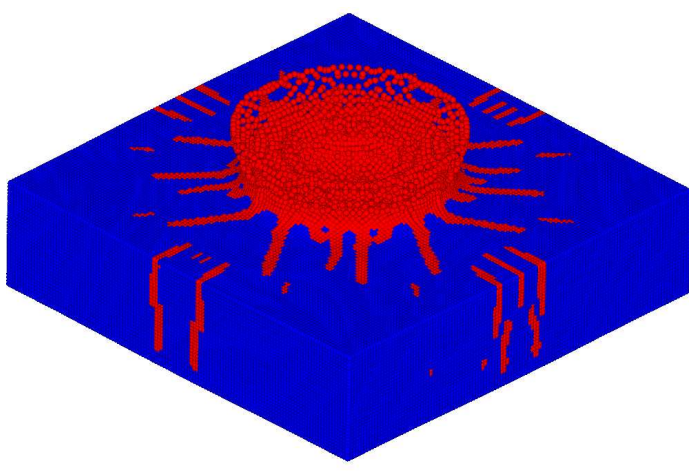

(a) $0.3 \mathrm{~ms}$

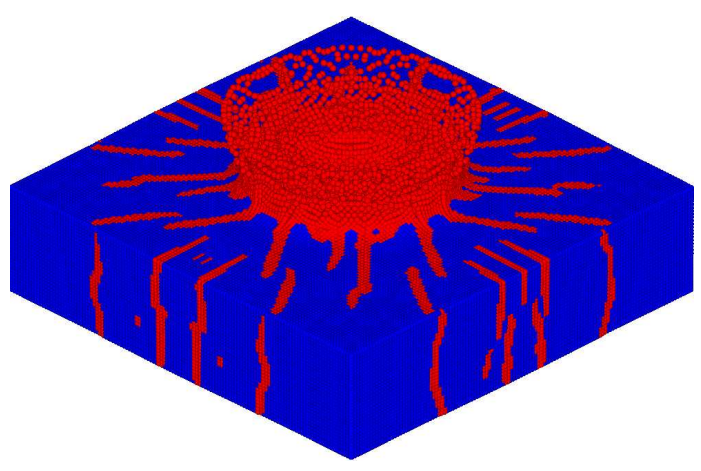

(c) $0.4 \mathrm{~ms}$

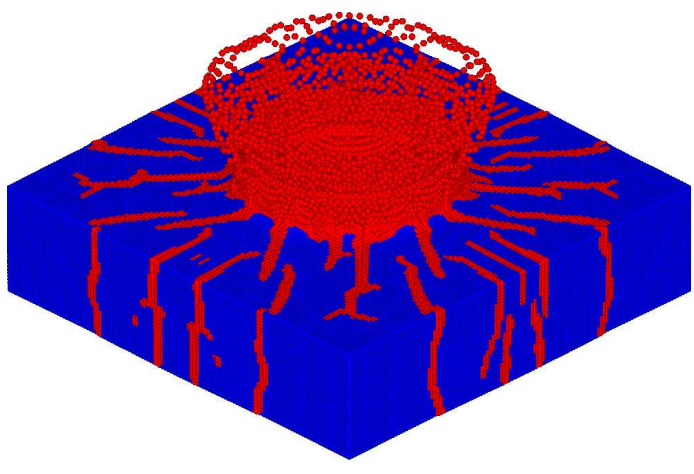

(e) $0.5 \mathrm{~ms}$

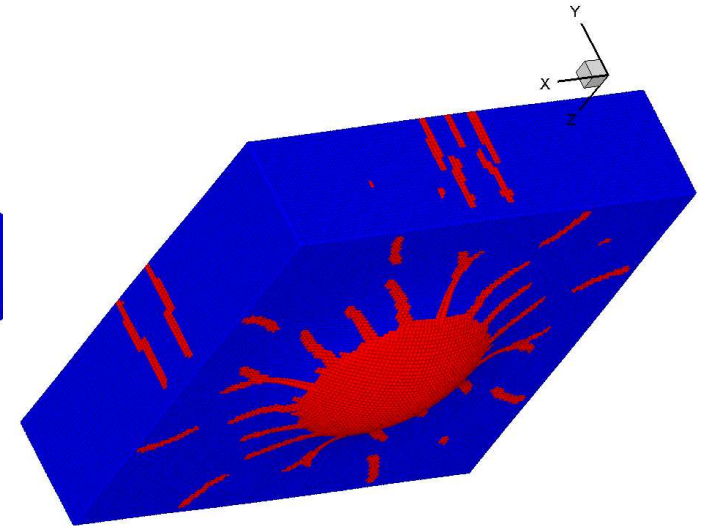

(b) $0.3 \mathrm{~ms}$

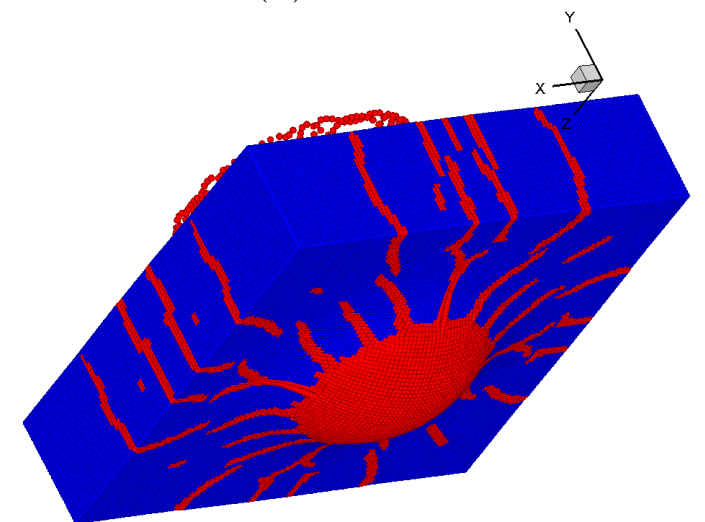

(d) $0.4 \mathrm{~ms}$

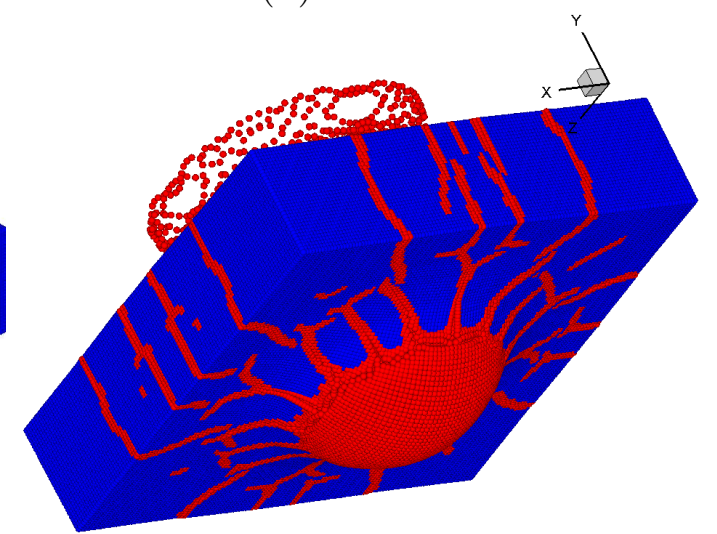

(f) $0.5 \mathrm{~ms}$

Fig. 29. Concrete slab O1 under explosive loading using approximately 2,100,000 particles, in red: cracked particles, in blue: uncracked particles

The time history of the overall dissipated energy is shown in figure 31 . In addition, we have performed a computation with approximately 1,000,000 particles. The results appear to converge with increasing numbers of particles.

In figure 33, results obtained for a computation using only an Eulerian kernel and without cracking particles as in standard SPH methods are presented. 


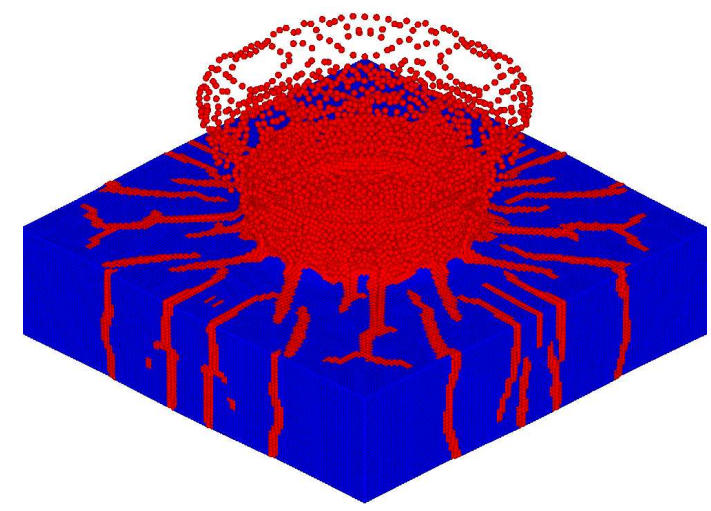

(e) $0.6 \mathrm{~ms}$

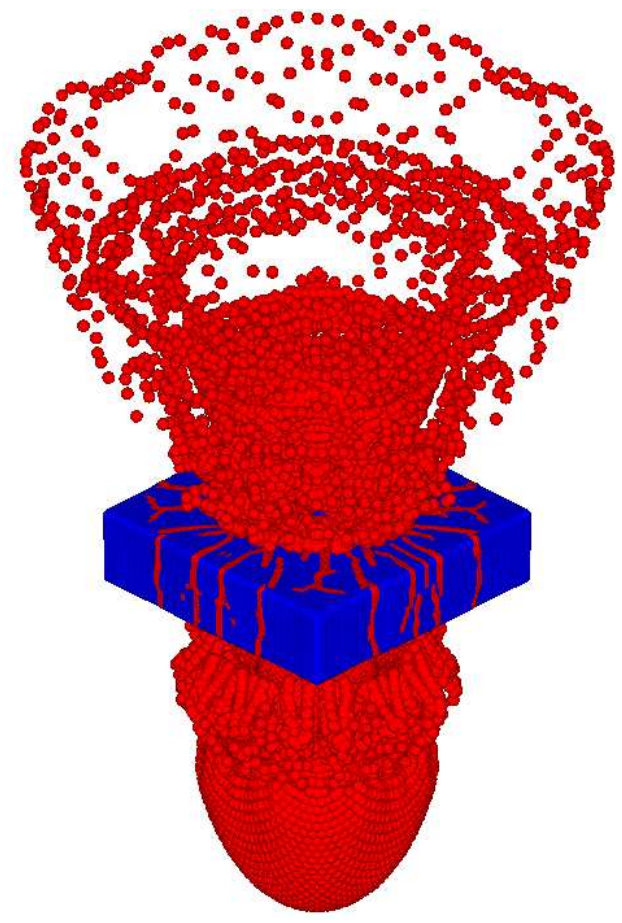

(a) $2.6 \mathrm{~ms}$

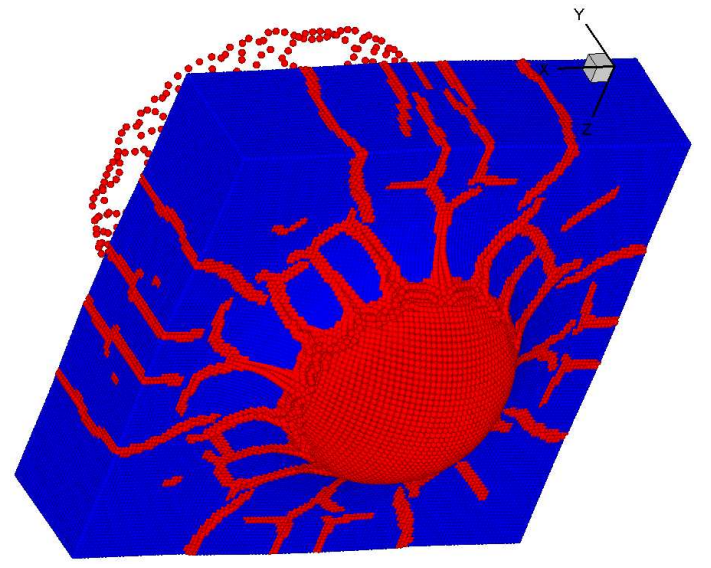

(f) $0.6 \mathrm{~ms}$

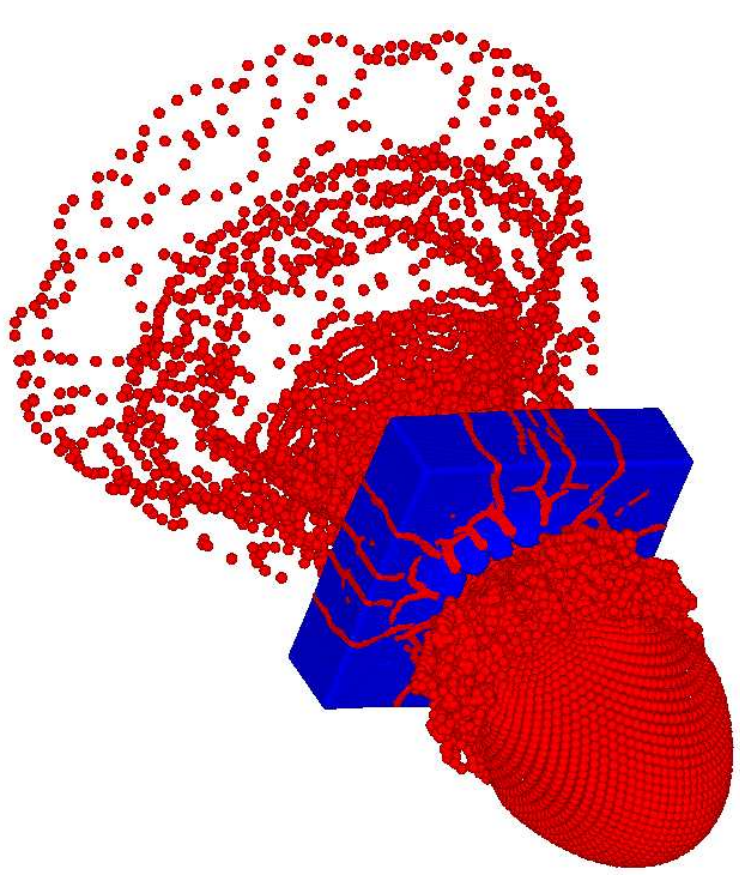

(b) $2.6 \mathrm{~ms}$

Fig. 30. Concrete slab O1 under explosive loading using approximately 2,100,000 particles, in red: cracked particles, in blue: uncracked particles

Numerical instabilities can be observed. These are manifested by excessively wide crack openings in combination with increasing damage of the entire structure when the computation proceeded further. The wavelike deformation of the concrete slab is also rather unrealistic for such a brittle material such as concrete. Computations with a purely Lagrangian kernel also became unstable later in the computation because of their inability to deal with the large deformations. With the present approach, we did not observe any evidence of numerical instabilities. Cracking stops at approximately $0.5 \mathrm{~ms}$ independent of the discretization and without further (artificial) damage evolution. Note, that the wide crack openings observed in the experiment, figure 32, occur at 


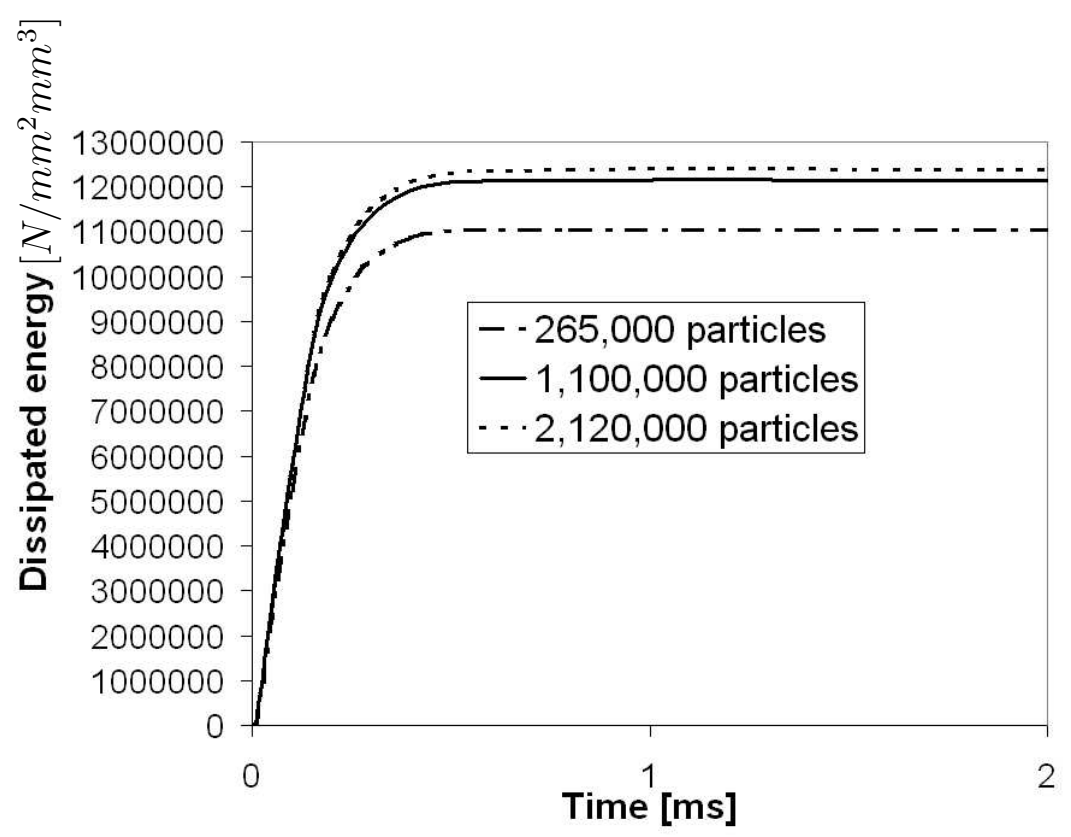

Fig. 31. Time history in the dissipated energy of the slab $O 1$
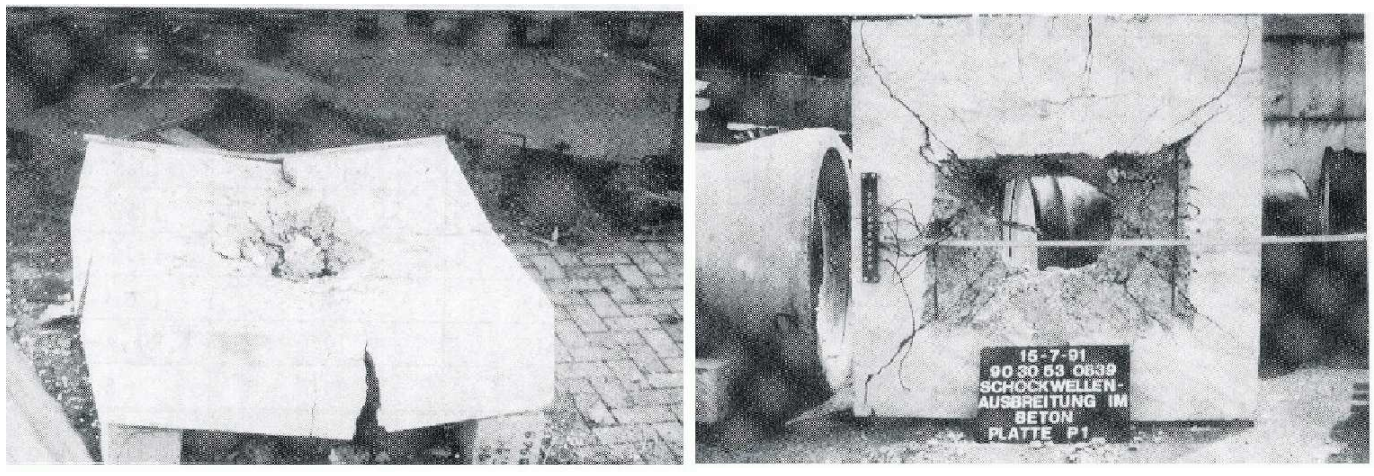

Fig. 32. Damage of the slab $O 1$ after the experiment a) top view, b) bottom view about 1 second and the simulation is stopped after a few miliseconds.

Next, we will focus on slab $H 1$. The deformation of the slab at different time steps and for different numbers of particles is shown in figure 34. Note that due to postprocessing problems only every second particle is plotted for the 4,000,000 particle simulation. For a better illustration, the slab is cut in half, so that the crack pattern and the crater diameter can be seen. After $0.5 \mathrm{~ms}$, the cracking process is completed and the slab starts to fragment. It can also be seen that under the crater and at the lower part of the slab, considerable damage occurs. Also the rest of the experimental crack pattern is reproduced well by the simulation. Note that the damage at the bottom and under the crater is internal damage.

The final deformation of slab $H 1$ is shown in figure 35 exemplarily for the 380,000 particle simulation. The crater diameter and the penetration depth is reproduced well. The results of the computation with the other models with 


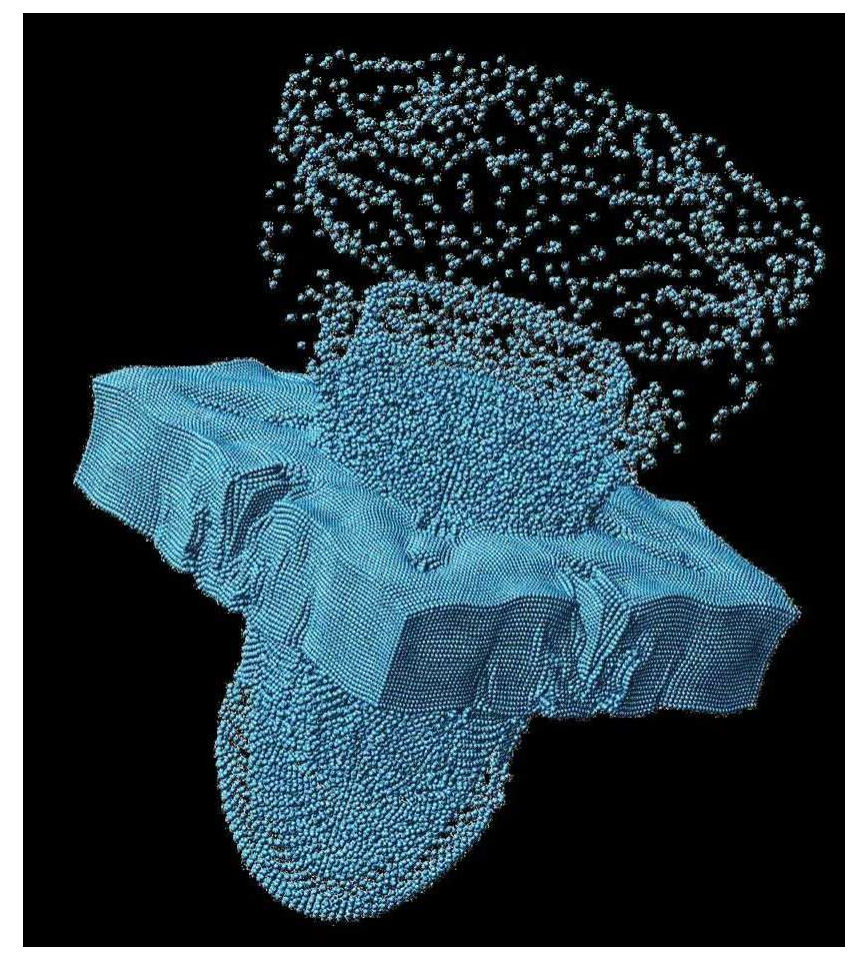

Fig. 33. Crack pattern of the concrete slab O1 using an Eulerian kernel

Table 7

Hydrostatic pressures in the concrete at different positions

\begin{tabular}{|c|c|c|c|}
\hline $\begin{array}{c}\text { Distance of the pressure } \\
\text { gauges to the loaded surface }\end{array}$ & Experiment & $\begin{array}{c}380,000 \text { particle } \\
\text { simulation }\end{array}$ & $\begin{array}{c}4.000,000 \text { particle } \\
\text { simulation }\end{array}$ \\
\hline $2 \mathrm{~cm}$ & $18,516 \mathrm{MPa}$ & $18,670 \mathrm{MPa}$ & $18,900 \mathrm{MPa}$ \\
\hline $7 \mathrm{~cm}$ & $1674 \mathrm{MPa}$ & $1890 \mathrm{MPa}$ & $1924 \mathrm{MPa}$ \\
\hline $17 \mathrm{~cm}$ & $74.4 \mathrm{MPa}$ & $95.5 \mathrm{MPa}$ & $98 \mathrm{MPa}$ \\
\hline $19.7 \mathrm{~cm}$ & $21.8 \mathrm{MPa}$ & $40.5 \mathrm{MPa}$ & $43 \mathrm{MPa}$ \\
\hline
\end{tabular}

different numbers of particles are very similar.

In table 7 , the maximum hydrostatic pressure at different positions in the concrete structure are given in terms of the distance from the midpoint of the explosive cone at the contact surface. The computed pressures match the experiment well. The influence of the refinement is not high for the range we used. 


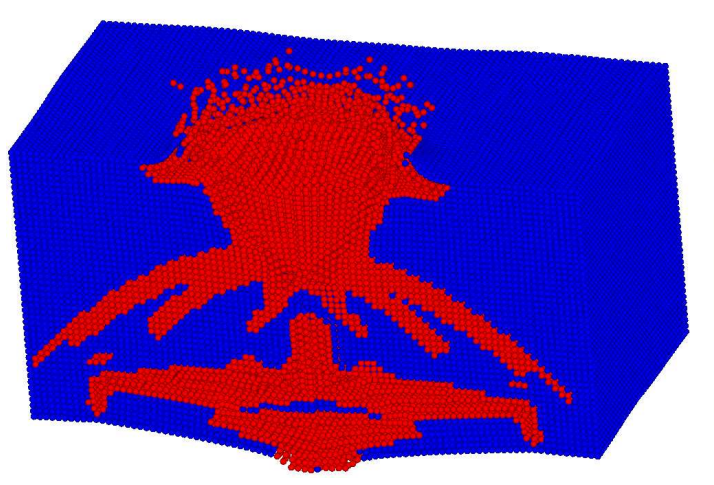

(a) $0.5 \mathrm{~ms}$

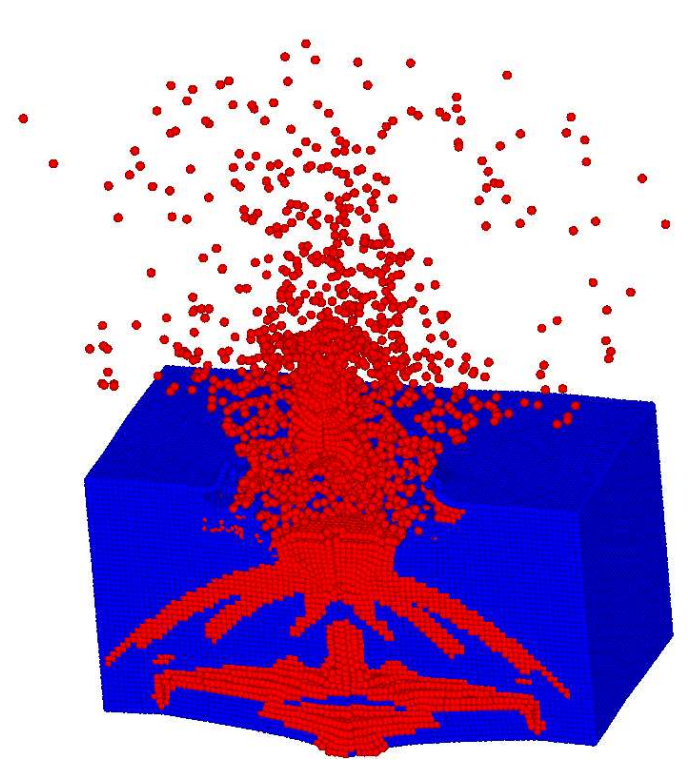

(c) $6.5 \mathrm{~ms}$

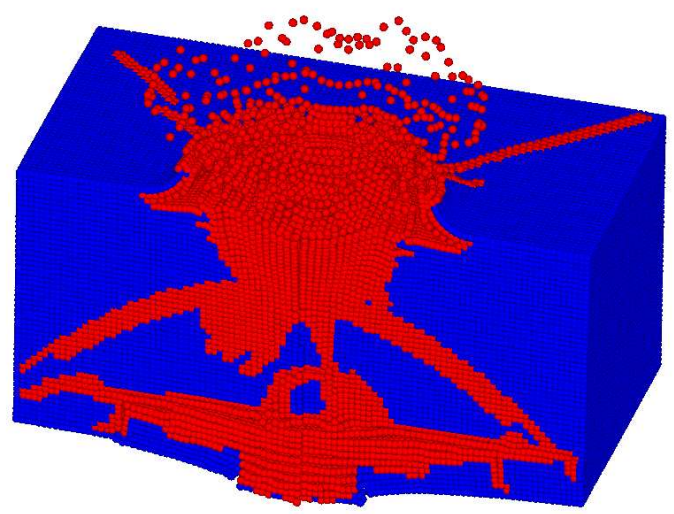

(b) $0.5 \mathrm{~ms}$

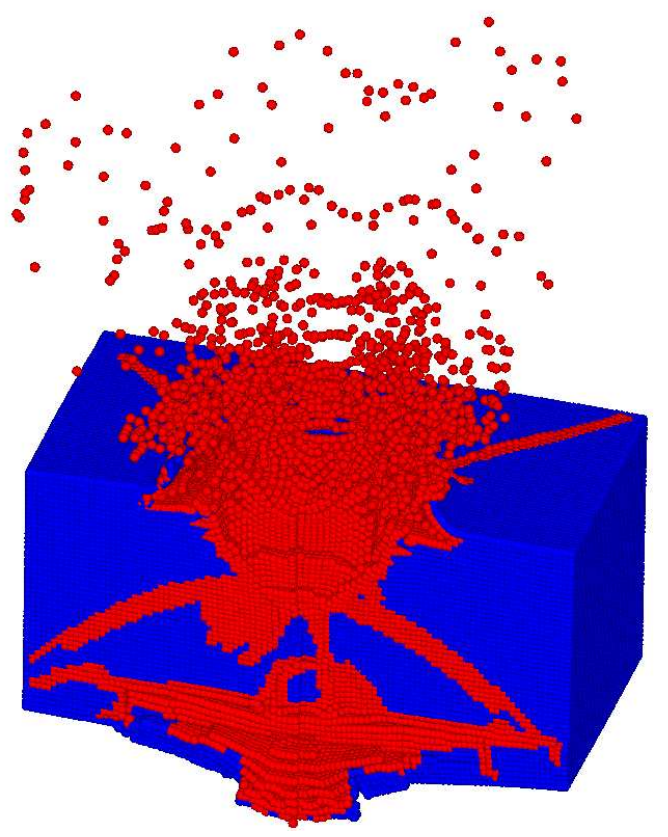

(d) $6.5 \mathrm{~ms}$

Fig. 34. Concrete slab H1 under explosive loading using at different time steps for: a) and c) approximately 380,000 particles; b) and d) 4.000.000 particles, in red: cracked particles, in blue: uncracked particles

\section{CONCLUSIONS}

A new method for treating crack growth by particle methods in three dimensions has been proposed. In this method, the crack is treated as a collection of cracked particles. At each cracked particle, a discontinuity along a plane is introduced normal to the failure direction. The method is applicable to large deformations. Different cracking criteria have been employed as rate dependent and rate independent constitutive and cohesive models.

The cracking criterion is checked independently at each particle. For a rate 


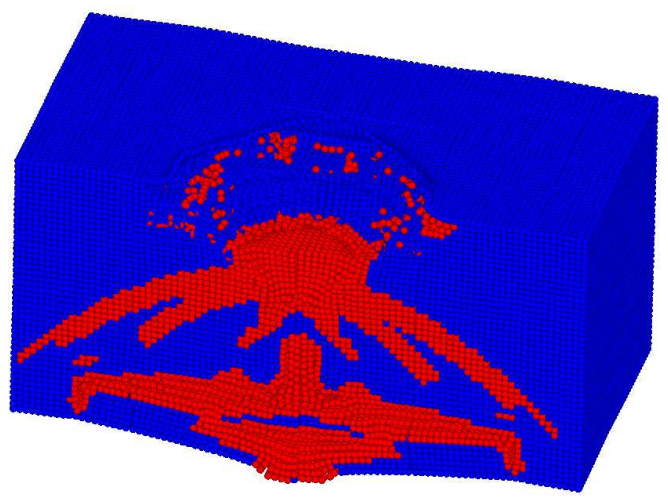

(a) 380,000 particles

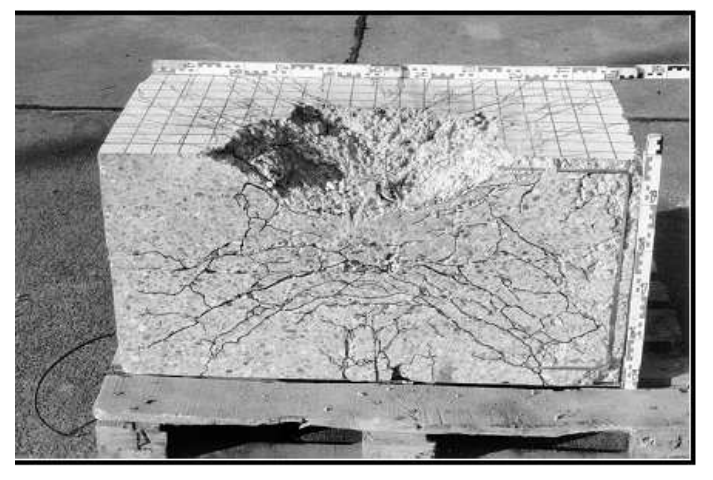

(b) Experiment

Fig. 35. Final crack pattern of concrete slab H1 compared to the experiment

independent material, we used loss of hyperbolicity while loss of material stability is used for a rate dependent constitutive law. Note, that loss of hyperbolicity and loss of material stability is checked by the same procedure. The method is able to treat the nucleation of cracks and complex patterns involving crack branching and crossing. This makes the methodology simpler than methods based on level sets or other explicit crack representations for complex patterns of fracture. Of course, this also limits the accuracy of the method somewhat.

An alternative to this method is the use of the visibility criterion with a set of planes centered at particles as in this method (see Belytschko and Tabbara [11]). The visibility criterion does not require extra degrees of freedom for cracked particles, which is an advantage over this method. However, checking the visibility criterion for a large number of cracked particles would be quite burdensome, even if the discrete cracks are circular discs in 3D.

In contrast to Rabczuk and Belytschko [36], the present method is based on a mixed Lagrangian-Eulerian kernel formulation. The Lagrangian kernel guarantees that material fracture occurs due to physical conditions. The switch to the Eulerian kernel around the fracture at a later stage guarantees that the method is stable also for extremly large deformations. We demonstrated this for two examples, sections 5.8 and 5.7.

We incorporated an h-adaptive approach that is useful for problems with a moderate number of cracks. A better resolution of the crack paths is achieved and computational cost is reduced. This is shown for two examples, section 5.4 and 5.6. The crack branching problem in section 5.6 was already solved in [36] in two dimensions. The corresponding 3D computation agrees very well with the two dimensional crack pattern. With the adaptive 3D computation, it is shown that the crack can be resolved much better with better resolved crack branches. For the non-planar crack propagation problem in section 5.4, we have shown that only adaptive computations lead to adequate solutions in 
an acceptable amount of time.

We also studied the method for two examples where an analytical solution is available, a 2D mode I-crack problem and a cube with a centered penny-shaped crack under uniaxial tension. We obtained global and local convergence where the relative error and the convergence rate is similar to the one obtained by the visibility method. It was also shown that a certain refinement around the crack front is necessary to obtain adequate results in terms of the local relative error.

The method performed quite well for several static and dynamic problems for which experimental results are available. Experimental crack patterns and damage were predicted quite accurately. Results compared well to experimental data, including highly dynamic events with extremely large deformations and strain rate effects such as concrete impact and contact detonations. Thus, in spite of the simplicity of the method, it appears to be extremely effective.

\section{ACKNOWLEDGEMENT}

The support of Office of the Naval Research under Grant N00014-03-0097 and the Army Research Office under Grant DAAD19-02-1-0339 is gratefully acknowledged.

\section{References}

[1] F. Armero and K. Garikipati. An analysis of strong discontinuities in multiplicative finite strain plasticity and their relation with the numerical simulation of strain localization in solids. International Journal of Solids and Structures, 33(20-22):2863-2885, 1996.

[2] M. Arrea and A.R. Ingraffea. Mixed-mode crack propagation in mortar and concrete. Technical Report 81-13, Dept. of Struct. Eng., Cornell University Ithaka, 1982.

[3] T. Belytschko and T. Black. Elastic crack growth in finite elements with minimal remeshing. International Journal for Numerical Methods in Engineering, 45(5):601-620, 1999.

[4] T. Belytschko, H. Chen, J. Xu, and G. Zi. Dynamic crack propagation based on loss of hyperbolicity and a new discontinuous enrichment. International Journal for Numerical Methods in Engineering, 58(12):18731905, 2003.

[5] T. Belytschko, J. Fish, and B. Englemann. A finite element method with embedded localization zones. Computer Methods in Applied Mechanics and Engineering, 70:59-89, 1988. 
[6] T. Belytschko, Y. Guo, W.K. Liu, and S.P. Xiao. A unified stability analysis of meshfree particle methods. International Journal for Numerical Methods in Engineering, 48:1359-1400, 2000.

[7] T. Belytschko, W. K. Liu, and B. Moran. Nonlinear Finite Elements for Continua and Structures. John Wiley and Sons, Chichester, 2000.

[8] T. Belytschko and Y.Y. Lu. Element-free galerkin methods for static and dynamic fracture. International Journal of Solids and Structures, 32:2547-2570, 1995.

[9] T. Belytschko, Y.Y. Lu, and L. Gu. Crack propagation by element-free galerkin methods. Engineering Fracture Mechanics, 51(2):295-315, 1995.

[10] T. Belytschko and M.O. Neal. Contact-impact by the pinball algorithm with penalty and lagrangian methods. International Journal for Numerical Methods in Engineering, 31:547-572, 1991.

[11] T. Belytschko and M. Tabbara. Dynamic fracture using element-free galerkin methods. International Journal for Numerical Methods in Engineering, 39(6):923-938, 1996.

[12] J. Bonet and S. Kulasegaram. Correction and stabilization of smooth particle hydrodynamics methods with application in metal forming simulations. International Journal for Numerical Methods in Engineering, 47 (6):1189-1214, 2000.

[13] J. Bonet and T. Lok. Variational and momentum preservation aspects of smooth particle hydrodynamic formulations. Computer Methods in Applied Mechanics and Engineering, 180(1-2):97-115, 1999.

[14] G. T. Camacho and M. Ortiz. Computational modeling of impact damage in brittle materials. International Journal of Solids and Structures, 33: 2899-2938, 1996.

[15] G.F. Carey. Copmutational Grids: Generation, Adaptation and Solution Strategies. Taylor and Francis, 1997.

[16] P. Devloo, T.J. Oden, and P. Pattani. An h-p adaptive finite element method for the numerical simulation of compressible flow. Computer Methods in Applied Mechanics and Engineering, 70(2):203-235, 1988.

[17] G.A. Dilts. Moving least square particle hydrodynamics i: Consistency and stability. International Journal for Numerical Methods in Engineering, 44:1115-1155, 2000.

[18] G.A. Dilts. Moving least square particle hydrodynamics ii: Conservation and boundaries. International Journal for Numerical Methods in Engineering, pages 1503-1524, 2000.

[19] J. Dolbow, N. Moes, and T. Belytschko. Discontinuous enrichment in finite elements with a partition of unity method. Finite Element Analysis and Design, 36(3):235-260, 2000.

[20] G. Etse and K. Willam. Failure analysis of elasto-viscoplastic material models. ASCE Journal of Engineering Mechanics, 125:60-69, 1999.

[21] M.L. Falk, A. Needleman, and J.R. Rice. A critical evaluation of cohesive zone models of dynamic fracture. Journal of Physics IV 11 (PR5), pages 43-50, 2001. 
[22] A. Gravouil, N. Moes, and T. Belytschko. Non-planar 3D crack growth by the extended finite element and level sets - part ii: Level set update. International Journal for Numerical Methods in Engineering, 53:25692586, 2002.

[23] N. Hermann. Experimentelle Erfassung des Betonverhaltens unter Schockwellen. PhD thesis, Institut fuer Massivbau und Baustofftechnologie, Universitaet Karlsruhe, 2002.

[24] Y. Krongauz and T. Belytschko. Consistent pseudo derivatives in meshless methods. Computer Methods in Applied Mechanics and Engineering, 146:371-386, 1997.

[25] P. Krysl and T. Belytschko. The element free galerkin method for dynamic propagation of arbitrary 3-D cracks. International Journal for Numerical Methods in Engineering, 44(6):767-800, 1999.

[26] J. Lemaitre. Evaluation of dissipation and damage in metal submitted to dynamic loading. Proceedings ICM 1, 1971.

[27] R. Loehner. Applied CFD Techniques: An introduction based on Finite Element Methods. John Wiley and Sons, LTD, 2001.

[28] H. Lu and J.S. Chen. Adaptive galerkin particle method. Lecture Notes in Computational Science and Engineering, 26:251-267, 2002.

[29] Y.Y. Lu, T. Belytschko, and M. Tabbara. Element-free galerkin method for wave-propagation and dynamic fracture. Computer Methods in Applied Mechanics and Engineering, 126(1-2):131-153, 1995.

[30] N. Moes, J. Dolbow, and T. Belytschko. A finite element method for crack growth without remeshing. International Journal for Numerical Methods in Engineering, 46(1):133-150, 1999.

[31] N. Moes, A. Gravouil, and T. Belytschko. Non-planar 3-D crack growth by the extended finite element method and level sets, parti: Mechanical model. International Journal for Numerical Methods in Engineering, 53 (11):2549-2568, 2002.

[32] J. Ockert. Ein Stoffgesetz fuer die Schockwellenausbreitung in Beton. $\mathrm{PhD}$ thesis, Institut fuer Massivbau und Baustofftechnologie, Universitaet Karlsruhe, 1997.

[33] M. Ortiz, Y. Leroy, and A. Needleman. Finite element method for localized failure analysis. Computer Methods in Applied Mechanics and Engineering, 61(2):189-214, 1987.

[34] M. Ortiz and A. Pandolfi. Finite-deformation irreversible cohesive elements for three-dimensional crack-propagation analysis. International Journal for Numerical Methods in Engineering, 44:1267-1282, 1999.

[35] T. Rabczuk and T. Belytschko. Application of meshfree particle methods to static fracture of reinforced concrete structures. submitted to International Journal of Fracture.

[36] T. Rabczuk and T. Belytschko. Cracking particles: A simplified meshfree method for arbitrary evolving cracks. International Journal for Numerical Methods in Engineering, 61(13):2316-2343, 2004.

[37] T. Rabczuk and T. Belytschko. Adaptivity for structured meshfree parti- 
cle methods in 2D and 3D. International Journal for Numerical Methods in Engineering, 63(11):1559-1582, 2005.

[38] T. Rabczuk, T. Belytschko, and S.P. Xiao. Stable particle methods based on lagrangian kernels. Computer Methods in Applied Mechanics and Engineering, 193:1035-1063, 2004.

[39] T. Rabczuk and J. Eibl. Simulation of high velocity concrete fragmentation using sph/mlsph. International Journal for Numerical Methods in Engineering, 56:1421-1444, 2003.

[40] P.W. Randles and L.D. Libersky. Recent improvements in sph modeling of hypervelocity impact. International Journal of Impact Engineering, 20:525-532, 1997.

[41] P.W. Randles and L.D. Libersky. Normalized sph with stress points. International Journal for Numerical Methods in Engineering, 48:14451462, 2000.

[42] E. Samaniego, X. Oliver, and A. Huespe. Contributions to the Continuum Modelling of Strong Discontinuities in Two-Dimensional Solids. $\mathrm{PhD}$ thesis, International Center for Numerical Methods in Engineering, Monograph CIMNE No. 72, Barcelona, Spain, 2003.

[43] H. Schuler. Experimentelle und numerische Untersuchungen zur Schaedigung von stossbeanspruchtem Beton. PhD thesis, Universitaet der Bundeswehr Mnchen, 2004.

[44] L.W. Swegle and D.A. Hicks. Smooth particle hydrodynamics stability analysis. Journal of Computational Physics, 116:123-134, 1995.

[45] M. Unosson and L. Nilsson. Projectile penetration and perforation of high performance concrete: Experimental results and macroscopic modelling. accepted in International Journal for Impact Engineering.

[46] L.P. Vila. On particle weighted methods and smooth particle hydrodynamics. Math. Models Methods Appl. Sci., 9(2):161-209, 1999.

[47] X.-P. Xu and A. Needleman. Void nucleation by inclusion debonding in a crystal matrix. Modell. Simul. Mater. Sci. Engng., 1:111-132, 1993.

[48] X.-P. Xu and A. Needleman. Numerical simulations of fast crack growth in brittle solids. Journal of the Mechanics and Physics of Solids, 42: 1397-1434, 1994.

[49] Y. You, J.S. Chend, and H. Lu. Filters, reproducing kernel and adaptive meshfree method. Computational Mechanics, 31:316-326, 2003.

[50] F. Zhou and J.F. Molinari. Dynamic crack propagation with cohesive elements: a methodolgy to address mesh dependence. International Journal for Numerical Methods in Engineering, 59(1):1-24, 2004. 